\title{
On the state-of-the-art of particle methods for coastal and ocean engineering
}

$\operatorname{AUTHOR}(\mathrm{S}):$

Gotoh, Hitoshi; Khayyer, Abbas

\section{CITATION:}

Gotoh, Hitoshi ...[et al]. On the state-of-the-art of particle methods for coastal and ocean engineering. Coastal Engineering Journal 2018, 60(1): 79-103

\section{ISSUE DATE:}

2018

URL:

http://hdl.handle.net/2433/235928

\section{RIGHT:}

This is an Accepted Manuscript of an article published by Taylor \& Francis in Coastal Engineering Journal on 26 February 2018, available online: http://www.tandfonline.com/10.1080/21664250.2018.1436243.; The full-text file will be made open to the public on 26 August 2019 in accordance with publisher's 'Terms and Conditions for Self-Archiving'.; この論文は出版社版でありません。引用の際には出版社版をご確認ご利用ください。; This is not the published version. Please cite only the published version. 


\title{
On the state-of-the-art of particle methods for
}

\section{coastal and ocean engineering}

\author{
Hitoshi Gotoh and Abbas Khayyer* \\ Department of Civil and Earth Resources Engineering, Kyoto University, \\ Katsura Campus, Nishikyo-ku, Kyoto 615-8540, Japan
}

*Corresponding author, Email: khayyer@particle.kuciv.kyoto-u.ac.jp 


\section{Abstract}

The paper aims at providing an up-to-date review on several latest advancements related to particle methods with applications in coastal and ocean engineering. The latest advancements corresponding to accuracy, stability, conservation properties, multi-phase multi-physics multi-scale simulations, fluid-structure interactions, exclusive coastal/ocean engineering applications and computational efficiency are reviewed. The future perspectives for further enhancement of applicability and reliability of particle methods for coastal/ocean engineering applications are also highlighted.

Keywords: particle methods, projection method, Smoothed Particle Hydrodynamics, Incompressible SPH, Moving Particle Semi-implicit, multi-phase, multi-physics, multi-scale, fluid-structure interactions, floating bodies, coastal hydrodynamics, coastal sediment transport, computational efficiency

\section{Introduction}

Due to their meshfree, Lagrangian nature, particle methods have been considered as potentially robust computational tools for a wide range of scientific and engineering applications. In particular, these new generation computational methods are intrinsically suitable to solve engineering problems characterized by complex physics involving moving boundaries and highly deformed interfaces such as those encountered in coastal and ocean engineering. During the past two decades, a great number of studies have been conducted on development and application of particle methods, including SPH [Smoothed Particle Hydrodynamics; Gingold and Monaghan, 1977] and MPS [Moving Particle Semi-implicit; 
Koshizuka and Oka, 1996] methods, for different fields of engineering including coastal/ocean engineering. These studies were mainly focused on enhancement of stability/accuracy as well as extension of applications.

Up to now, many studies have portrayed potential applicability of particle methods in coastal/ocean engineering. Examples include simulations related to wave breaking [e.g. Farahani and Dalrymple, 2014], wave overtopping [e.g. Shao et al., 2006], wave run-up [e.g. Shadloo et al., 2015], wave impact [e.g. Altomare et al., 2015], wave-induced nearshore circulation system [Farahani et al., 2014], violent sloshing [e.g. Gotoh et al., 2014], oil spilling [e.g. Violeau et al., 2007; Duan et al., 2017], green water on ships [e.g. Le Touzé et al., 2010], sediment transport [e.g. Gotoh and Sakai, 2006] and fluid-structure interactions [e.g. Colagrossi et al., 2015; Wei et al., 2015]. Several important key features of particle methods for coastal/ocean engineering applications have been highlighted in a number of review papers [Koshizuka, 2011; Shadloo et al., 2016; Gotoh and Khayyer, 2016; Violeau and Rogers, 2016; Gotoh and Okayasu, 2017].

Particle methods applied for free-surface fluid flows can be categorized into two categories of weakly compressible and incompressible ones. The weakly compressible particle methods such as Weakly Compressible SPH [WCSPH; e.g. Colagrossi and Landrini, 2003; Dalrymple and Rogers, 2006] or Weakly Compressible MPS [WCMPS; e.g. Tayebi and Jin, 2015] methods solve an appropriate equation of state in a fully explicit form. The incompressible particle methods such as MPS or Incompressible SPH [ISPH; e.g. Shao and Lo, 2003] methods solve a Poisson Pressure Equation (PPE) through a Helmholtz-Leray decomposition [Foias et al. 2001] and application of Chorin's projection 
method [Chorin, 1968]. Hence, they can be referred to as projection-based particle methods.

Several comparative studies have investigated the performance of ISPH with respect to WCSPH [e.g. Lee et al., 2008]. In general, projection-based particle methods are expected to possess higher levels of accuracy, especially in terms of pressure calculation and volume conservation, provided that accurate/consistent differential operator models are applied along with careful implementations of boundary conditions [Gotoh and Okayasu, 2017].

This paper aims at reviewing several latest advancements made in the field of particle methods, with applications in coastal/ocean engineering. The ongoing studies and existing challenges will be also highlighted. The latest advancements correspond to accuracy, stability and conservation properties, multi-phase multi-physics multi-scale simulations, Fluid-Structure Interactions (FSI), exclusive coastal/ocean engineering applications (floating bodies, coastal hydrodynamics, coastal sediment transport) and computational efficiency.

\section{Latest advancements}

\subsection{Accuracy, stability, conservation properties}

The governing equations corresponding to free-surface fluid flows are the continuity and Navier-Stokes equations written in a continuous framework as follows: 


$$
\begin{aligned}
& \frac{1}{\rho} \frac{D \rho}{D t}+\nabla \cdot \boldsymbol{u}=0 \\
& \frac{D \boldsymbol{u}}{D t}=-\frac{1}{\rho} \nabla p+\boldsymbol{g}+v \nabla^{2} \boldsymbol{u}
\end{aligned}
$$

where $\boldsymbol{u}$ denotes particle velocity vector; $t$ stands for time; $\rho$ represents fluid density; $p$ symbolizes pressure; $\boldsymbol{g}$ signifies gravitational acceleration vector and $v$ represents laminar kinematic viscosity. It should be noted that Eq. (1) is written in the form of a compressible flow. In general, particle-based simulations of the incompressible fluid flows have been carried out in two different frameworks of fully-explicit WCSPH and semi-implicit projection-based methods such as MPS and Incompressible SPH (ISPH). In the framework of WCSPH, the relation between pressure and density is established via application of an equation of state, in a general form as:

$$
p=p(\rho)
$$

In the literature, various forms of equations of state have been employed e.g. stiffened equation of state (Eq. 4) [Monaghan, 2012] or linear form of equation of state based on Taylor expansion of pressure around reference density $\rho_{0}$ (Eq. 5) [Antuono et al., 2010].

$$
\begin{aligned}
& p=p_{0}+\frac{c_{s} \rho_{0}}{\gamma}\left(\left(\frac{\rho}{\rho_{0}}\right)^{\gamma}-1\right) \\
& p=p_{0}+c_{s}^{2}\left(\rho-\rho_{0}\right)
\end{aligned}
$$


where $\rho_{0}$ signifies the reference density, $p_{0}$ denotes the ambient pressure, and $c_{s}$ is the speed of sound.

In projection-based particle methods, pressure is obtained based on enforcing the constraint of incompressibility, i.e. $D \rho / D t$ equal to zero (Eq. 1), at each particle and at each calculation time step through application of Helmholtz-Leray decomposition and within a prediction-correction procedure. At the end of each time step a Poisson Pressure Equation PPE) is solved as:

$$
\left\langle\nabla^{2} p_{k+1}\right\rangle_{i}=\frac{1}{\Delta t}\left(\frac{D \rho}{D t}\right)_{i}^{*} ; \rho=m \sum_{j} w\left(\left|\boldsymbol{r}_{i j}\right|\right)=m \sum_{j} w_{i j} \quad ; \quad \boldsymbol{r}_{i j}=\boldsymbol{r}_{j}-\boldsymbol{r}_{i}
$$

where $\Delta t$ corresponds to calculation time step, $k$ represents calculation step number, $i$ and $j$ denote target and neighboring particles, respectively, $m$ signifies particle mass, $w_{i j}$ symbolizes kernel function and $\boldsymbol{r}_{i j}$ denotes relative position vector of neighboring particle $j$ with respect to target particle $i$. In Eq. (6), the superscript * corresponds to the pseudo time step $k+1 / 2$ (the end of prediction step of the two-step prediction-correction solution process).

There are several different categories of schemes presented in the context of particle methods to achieve more accurate solutions to the abovementioned equations. In addition, there are several important studies on conservation properties of particle methods including energy, momentum and volume conservations, that will be briefly reviewed in this section. 
In the context of Weakly Compressible SPH (WCSPH), the so-called $\delta$-SPH [Antuono et al., 2010 and 2012], Riemann SPH [Inutsuka 2002; Gao et al., 2012; Rafiee et al., 2012; Cercos-Pita et al., 2016] and higher-order accurate SPH [e.g. Colagrossi et al., 2011; Le Touzé et al., 2013; Litvinov et al., 2015; Meringolo et al., 2017] have been proposed for enhanced levels of accuracy and stability. Le Touzé et al. [2013] investigated different numerical aspects in simulation of free surface flows by using WCSPH, where the effects of numerical errors in creation of acoustic wave frequencies in the pressure field and the choice of sound speed were discussed. In addition, Le Touzé et al. [2013] highlighted the necessity of utilization of several numerical schemes (artificial viscosity, corrective term for tensile stability and Periodic density reinitialization) for robustness and accuracy of the WCSPH method.

In the framework of projection-based particle methods, refined differential operator models have been presented to achieve a more accurate projection by using higher-order and more consistent schemes as described in the review paper by Gotoh and Khayyer [2016]. Several studies have been conducted on comparing the stability/accuracy of ISPH with respect to WCSPH in simulation of $2 \mathrm{D}$ free-surface fluid flows, e.g. Rafiee et al. [2012] and Zheng et al. [2014]. Jian et al. [2016] carried out a comparative study on 3D WCSPH and ISPH methods in terms of numerical efficiency and accuracy, in simulation of dam-break flows impacting fixed and movable structures. Zheng et al. [2017a] compared several consistency-related corrections for pressure gradient calculation to achieve a more accurate estimation of wave impact pressure. 
For both WCSPH and projection-based particle methods (e.g. ISPH, MPS), a distinct category of schemes, the so-called particle regularization schemes, has been developed to enhance the regularity of particle distributions that would improve the accuracy, stability and convergence properties. One of the well-known and robust particle regularizing schemes with extended application to free-surface flows, corresponds to the so-called Particle Shifting (PS) scheme initially proposed by Xu et al. [2009] and then extended to a generalized form by Lind et al. [2012]. The PS scheme is founded on Fick's diffusion law and a Taylor-series expansion for evaluation of particle quantities in new positions. This scheme has been applied in both WCSPH [e.g. Sun et al., 2017] and ISPH [e.g. Leroy et al., 2015] frameworks.

As another particle regularization scheme, the so-called DS (Dynamic Stabilization) [Tsuruta et al., 2013] scheme tends to regularize particle distributions by generating minimum required inter-particle repulsive force to keep them regularly spaced. This repulsive force is generated in a radial and anti-symmetric, and thus momentum conservative manner. Khayyer et al. [2017a] carried out a comparative investigation on accuracy and conservation properties of the DS and PS schemes, in simulations of both internal and free-surface flows in the context of ISPH method, where the superior momentum conserving property of DS with respect to PS was shown numerically. The PS was found to have relatively higher energy conservation properties and this indicated the need for proposal of an enhanced DS. Khayyer et al. [2017a] also proposed an improved shifting scheme referred to as Optimized PS (OPS) for accurate and consistent implementation of particle shifting for free-surface flows. 
In the framework of WCSPH, Adami et al. [2013] proposed a particle velocity correction together with a consistent additional term in the momentum equation in order to take the required modification of the advection velocity into account. The scheme was proven to be effective in enhancing the accuracy and stability of internal flows. However, extensions to free-surface flows do not appear to be straightforward. Oger et al. [2016b] proposed a specific transport velocity within an ALE formalism, where the method was shown to be robust and accurate for both internal and free-surface flows. Sun et al. [2017] proposed an enhanced version of $\delta$-SPH scheme with a particle shifting technique as well as special treatment for particles that are close to the free-surface region. The $\delta$-SPH has been further enhanced by implementation of a so-called TIC (Tensile Instability Control) for stable/accurate simulation of high Reynolds number flows [Sun et al., 2018].

As for recent advancements related to conservation properties, Antuono et al. [2015] investigated the energy balance in the $\delta$-SPH [Antuono et al. 2010 and 2012]. They focused on the dissipative effect of a diffusive operator inside the continuity equation through performing a number of test cases. They eventually concluded that the dissipative effect corresponding to diffusive operator is generally small and is activated when spurious high-frequency acoustic components are stimulated. Cercos-Pita et al. [2016] conducted a thorough study on several consistency conditions for a set of different existing diffusive terms that are incorporated in the mass conservation equation. Also equivalence between the diffusive term of the mass variation rate equation, used in the SPH Riemann solver, and the diffusive term in the conservation of mass equation, used in $\delta$-SPH was proved. All the considered diffusive terms by Cercos-Pita et al. [2016] were shown to bring about a 
challenge related to Thermodynamic consistency close to the boundaries where a physical energy dissipation could not be guaranteed.

Cercos-Pita et al. [2017] studied the energy conservation properties of SPH in the presence of fluid-solid interactions. Consistent solid boundary conditions were set by extensions of the pressure and velocity fields on the solid particles. It was shown that, in the presence of solid particles, the energy equation of the particle system includes some extra terms depending on the pressure-velocity field extensions. It was found that these terms are globally dissipative being thus consistent with Second Law of Thermodynamics, yet, conjectured to result in extra dissipations.

Meringolo et al. [2017] presented a filtering technique based on Wavelet Transform for high-frequency acoustic noises associated with weakly compressible SPH. They highlighted that in cases of violent impact problems, special care should be devoted to the filtering procedure, since removal of the acoustic component is not always possible depending on the continuity or discontinuity of mechanical energy.

In the context of projection-based particle methods, the skew-adjointness of gradient and divergence operators is required for an exact projection [Cummins and Rudman, 1999] which is also a necessity for an exact energy conservation. Violeau [2012] highlighted the skew-adjointness of gradient and divergence operators for energy conservation. Khayyer et al. [2017b] conducted a detailed study on energy conservation properties of projection-based particle methods, i.e. MPS and ISPH and highlighted the significance of Taylor-series consistent pressure gradient models and enhancing effect of the consistency-related Gradient Correction (GC) scheme in providing enhanced energy conservation. 
$\mathrm{Xu}$ and Lin [2017] proposed a new two-step projection ISPH method by including calculated pressure fields at both current $(t)$ and subsequent $(t+1)$ time steps in the projection process. Accordingly, pressure gradients at both current and future time steps were utilized with an equal weight in calculation of an updated intermediate velocity prior to obtaining particle positions for future time step $t+1$. The updated intermediate velocity and pressure fields were then interpolated using a second-order consistent (C2) kernel-based interpolation to result in the final velocity/pressure fields at time step $t+1$. The model was verified in simulation of a few test cases including linear and solitary wave propagations in constant water depth and nonlinear wave transformation over a submerged breakwater. The new two-step projection ISPH was shown to possess superior energy conservation properties with respect to the traditional two-step projection-based one. Also, in the context of projection-based particle methods, the concept of Space Potential Particles (SPP) was developed by Tsuruta et al. [2015] by introducing a potential in void space to improve the local volume conservation and reproduce physical motions of particles in the vicinity of free-surface through a particle-void interaction.

\subsection{Multi-phase, multi-physics, multi-scale simulations}

Multi-phase flows, or flows characterized by distinct physical phases, are widely encountered in a variety of problems associated with coastal/ocean engineering. In general, multi-phase flows in coastal/ocean engineering comprise of multi-phase solid-liquid flows and multi-phase liquid-gas flows. Presence of multiple simultaneous physical phenomena is another important matter in coastal/ocean engineering problems, leading to so-called multi-physics simulations. Another important issue regarding flows in coastal/ocean 
engineering corresponds to the different physical scales being involved ranging from micro to macro scales. From computational aspects, development of adaptive codes that can take in different computational resolutions and thus can reliably model different physical scales, would be of crucial importance. In this section, we review the latest advancements related to multi-phase solid-liquid flows, multi-phase gas-liquid flows, as well as multi-physics and multi-scales simulations.

\subsubsection{Multi-phase solid-liquid flows}

Typical examples of multiphase solid-liquid flows encountered in coastal/ocean engineering include sediment transport and scouring that are of great importance, for instance, in coastal morphological computations and design of coastal infrastructures. In view of computational difficulties involved in tracking movable interfaces as well as challenges in proper handling of interface discontinuities and appropriate implementation of interface boundary conditions, simulation of multi-phase solid-liquid flows has been among the challenging problems in coastal/ocean engineering.

In the context of particle methods, treatment of solid-liquid flows for sediment transport is usually made within two distinct groups, namely, integrated continuum-based modeling and continuum-discrete modeling where continuum fluid models are coupled with discrete soil models. As for integrated continuum-based methods, a continuum particle-based model for fluid is integratedly combined with another continuum particle-based model for soil through incorporation of an appropriate soil model, e.g. by treating soil as a non-Newtonian fluid [e.g. Razavitoosi et al., 2014] or an elastic-viscoplastic material [e.g. Ghaïtanellis et al., 2018]. 
In the framework of integrated particle-based modeling of multi-phase solid-liquid flows, Gotoh and Sakai [2006] presented a set of coastal engineering-related results by an integrated liquid-solid MPS-based method. Among other works, Ghaïtanellis et al. [2018] coupled an elastic-viscoelastic model with SPH to model non-cohesive sediment transport. A Drucker-Prager's criterion was used as the yielding criterion below which the deviatoric stresses were calculated based on a linear elastic model. A shear-thinning non-Newtonian fluid model was set for the sediments beyond the yield stress. Bui and Nguyen [2017] coupled SPH for pore-fluid (incompressible) with an elasto-plastic solid model in a deformable porous medium.

As for continuum-discrete modeling, one of the most common applications of particle-based methods in modeling solid-liquid interactions corresponds to the coupling in between SPH/MPS and Discrete Element Method (DEM) methods. Ren et al. [2013] developed a 2D DEM-SPH model for simulation of wave-structure interaction on a slope and evaluated the hydraulic stability of the armor blocks on rubble-mound breakwaters. Canelas et al. [2016] integrated the advanced contact mechanics theories with SPH and presented a so-called DCDEM (Distributed Contact Discrete Element Method)-SPH for resolved, accurate simulations of fluid-solid phases.

Harada et al. [2017] applied DEM-MPS coupling model for simulation of swash beach process and highlighted the significance of seepage flow in this process. Fig. 1(a) presents the computational domain corresponding experiments by Ma et al. [2004]. Both fluid and DEM particle sizes are considered as $d_{0}=5.0 \mathrm{E}-3 \mathrm{~m}$. The Young's modulus and Poisson's ratio are set as $E=5.0 \mathrm{E}+6 \mathrm{~Pa}$ and $v_{\text {pos }}=0.3$, respectively. Permeable bed surface is 
composed of gravel particles with $0.15 \mathrm{~m}$ thickness $\left(h_{e}=0.15 \mathrm{~m}\right)$ in Fig. 1(b-c), and gravel and plastic particles with $0.20 \mathrm{~m}$ thickness $\left(h_{e}=0.15 \mathrm{~m}\right)$ in Fig. $\mathbf{1}(\mathbf{d}-\mathbf{e})$, corresponding to experiment by Ma et al. [2004]. The frictional coefficient, $\mu$, is set 0.577 for gravel and 0.3 for plastic particles. Fig. 1(b-c) illustrates a qualitative comparison of exfiltration process between simulation and experiment by Ma et al. [2004]. As shown in this figure, the exfiltration process of dye is reproduced well by DEM-MPS coupling model, which validates the accuracy of DEM-MPS coupling model for reproduction of seepage flow. Fig. 1(d-e) portrays typical snapshots of particles together with horizontal velocity distributions, where DEM-MPS coupling model is shown to have a potential in simulating the movement of bed surface as well as to reproducing the seepage flow inside a permeable bed.

Indeed, the choice of selection of either integrated continuum-based or continuum-discrete modeling would depend on the problem of interest and the behavior of targeted soil. For instance, as for granular materials, discreteness of the system and consideration of rotational degrees of freedom may become crucially important and in such a case, DEM can be considered as an appropriate tool provided that accurate prediction of input parameters are made [Coetzee, 2017]. A challenge in continuum-discrete modeling would be related to precise implementation of a mathematically sound and physically consistent coupling in between continuum-based fluid model and discrete soil model. As for integrated continuum-based models, selection of appropriate constitutive equations that can well describe the rheological properties of granular materials, for instance, would be of significant importance. An integrated continuum-based approach is expected to provide a more manageable framework for accurate implementation of a mathematically sound, 
physically consistent coupling, with more accurate imposition of fluid-soil interface boundary conditions.

\subsubsection{Multi-phase liquid-gas flows}

The liquid-gas multi-phase flows are of great importance in many significant phenomena in coastal/ocean engineering such as hydrodynamic slamming, violent sloshing and violent aerated wave impacts. Numerical simulation of the mentioned liquid-gas multiphase flows is among the most challenging topics for coastal engineers. In fact, the complexities involved in multi-phase problem mainly arise from the sharp/abrupt density variation at the phase interface that would lead to a mathematical discontinuity of density and accordingly a discontinuous pressure gradient field. At the same time, for multi-phase flows characterized by large deformations of phase interfaces, particle methods provide a substantial potential of flexibly/conservatively handling sharp interfaces without a need for an interface capturing scheme (e.g. VOF, Level-set, etc.). In addition, compared to mesh-based methods, particle methods can provide superior results for violent multi-phase flows characterized by air entrapment/entrainment due to their meshfree Lagrangian features. Indeed, for a precise modeling of multi-phase liquid-gas flows in the context of coastal/ocean engineering, e.g. estimation of energy dissipation corresponding to breaking waves [Mori and Kakuno, 2008, Stagonas et al., 2011], an appropriate surface tension model should be incorporated.

There have been several attempts for development of accurate particle-based multiphase methods that can deal with mathematical discontinuity of density at the phase interfaces. In most of the conducted studies, specific numerical treatments were considered at the phase 
interface in order to improve the stability. Such numerical treatments include artificial repulsive pressure force [Monaghan and Rafiee, 2013], artificial surface tension force [Colagrossi and Landrini, 2003], particle shifting scheme [Lind et al., 2015 and 2016b; Mokos et al., 2017] or density smoothing schemes [Gotoh and Khayyer, 2016]. In the context of projection-based particle methods, Khayyer and Gotoh [2016] developed an extended version of ECS scheme to minimize the projection-related errors in an air-water compressible-incompressible multi-phase calculation for wave slamming.

\subsubsection{Multi-physics simulations}

In many problems encountered in coastal/ocean engineering multiple simultaneous physical phenomena exist. Examples include, FSI (Fluid-Structure Interactions), coastal-related heat diffusion problems such as diffusion of heated effluents and fluid flow interactions with porous media.

One of the most interesting multi-physics applications of particle-based methods in coastal engineering corresponds to fluid flow interactions with porous media, which is of great importance in evaluation of stability and reliability of several important coastal infrastructures. Although in some studies, interactions between fluid flow and porous media have been modeled in pore-scale [e.g. Ovaysi and Piri, 2012], simulation of fluid flow interactions with porous media from macroscopic viewpoint is of particular interest. In the macroscopic modeling, the effects of porous media can be considered by including linear and non-linear resisting force terms in the momentum equation [e.g. Ren et al., 2014; Gui et al., 2015]. 
In the context of projection-based methods, Shao [2010] presented an ISPH-based model for simulation of wave interactions with porous media. The effect of porous media was represented by additional friction force terms in the Navier-Stokes equation. Khayyer et al. [2017c] developed an Enhanced ISPH-based numerical method for simulation of fluid flow interactions with saturated porous media. Taking advantage of a set of enhanced schemes [Gotoh and Khayyer, 2016; Gotoh and Okayasu, 2017], the ISPH-based method provided an accurate representation of fluid flow-porous media interface without any special boundary treatment.

More complexities arise in simulation of fluid flow interactions with unsaturated porous media. Peng et al. [2017] considered the concept of volume fraction in the framework of so-called mixture theory, in order to provide a more rigorous representation of interface treatment. The approach by Peng et al. [2017] can be extended to reproduce fluid flow interactions with unsaturated porous media. The concept of variations of particle volume was incorporated in a multi-resolution MPS method by Ikari et al. [2017] for fluid flow interactions with unsaturated porous media. In the study by Bui and Nguyen [2017], the interaction with porous media as well as the deformation of porous media were well reproduced by incorporating elastic-plastic behavior of porous media.

In another interesting study related to multi-physics simulations, Kazemi et al. [2017] developed a 2D SPH-based method for simulation of depth-limited open channel turbulent flows over hydraulically rough bed. The model was founded on the solution of Navier-Stokes equations with incorporation of a shear stress term corresponding to the bed form-induced drag, along with a turbulence shear stress term obtained by modified version 
of SPS model. A mixing-length approach was proposed instead of standard Smagorinsky method for calculation of eddy viscosity in the modified SPS turbulence model.

\subsubsection{Multi-scale simulations}

The need for huge amount of computational power, in terms of both computational processing, i.e. Central Processing Unit (CPU) or Graphic Processing Unit (GPU), as well as computational memory, i.e. Random Access Memory (RAM), has been always among the great challenges in particle-based simulations of complex phenomena in coastal/ocean engineering, especially for 3D calculations. On the other hand, most critical phenomena for which spatial resolution refinements are required usually occur inside a limited region of the whole computational domain. Hence, development of multi-resolution schemes for implementation of particle methods on a multi-scale computational system would lead to considerable improvement of computational efficiency as well as adaptivity.

In the framework of SPH, dynamic particle refinement algorithms in two and three dimensions are developed and validated by Vacondio et al. [2013] and Vacondio et al. [2016], respectively. In the mentioned studies, coarse particles in low resolution area, namely "mother particles", are to be split into sets of finer particles namely "daughter particles", when mother particles enter high resolution area. Xiong et al. [2013] implemented Adaptive Particle Splitting and merging (APS) technique for SPH method on GPU. Particle splitting and merging were set based on a prescribed criterion. $\mathrm{Hu}$ et al. [2017b] further enhanced the APS technique by utilizing higher order interpolation scheme in merging process and applied the multi-resolution SPH-based simulation of 
fluid-structure interactions. Sun et al. [2017] proposed an algorithm for repositioning the particles in multi-resolution simulations by using a generalized particle-shifting scheme.

As for application of multi-resolution schemes in projection-based particle methods, Tsuruta et al. [2016] developed a multi-resolution scheme for implementation of MPS-based model on sets of particles with multi-scale refinements. Tang et al. [2016] developed improved multi-resolution scheme by utilizing averaged influence length as well as employing a modification parameter in weight averaging process. Shibata et al. [2017] proposed overlapping particle technique, based on which separated domains of high and low resolutions were connected to each other by considering an overlapping region.

\subsection{Fluid-structure interactions}

A great number of substantial problems associated with coastal/ocean engineering are entangled with highly interactive systems including violent fluid flows and structures. Interactions between wind induced waves and coastal structures, hydrodynamic slamming of marine vessels, tsunami/storm surge impact on onshore structures are instances of essential FSI challenges in the field of coastal/ocean engineering.

With respect to the intrinsic properties of most challenging FSI problems in coastal/ocean engineering (e.g. existence of violent free-surface flows, presence of large/abrupt hydrodynamics loads and consequently large structural deformations), particle methods e.g. SPH or MPS have the merits of being robust candidates for computational modeling of these important phenomena. Therefore, in recent decades, many efforts have been devoted to development of particle-based methods for modeling the FSI problems related to coastal and ocean engineering. 
In general, fluid-structure interactions can be divided into two major categories of fluid flow interactions with rigid structures and deformable ones. In the following subsections, the state-of-the-art of research on particle-based simulation of fluid flow interactions with rigid and deformable structures is described.

\subsubsection{Fluid flow interactions with rigid structures}

As for fluid flow interactions with rigid structures, Gomez-Gesteira et al. [2005] simulated wave overtopping on the decks of fixed offshore platforms and ships by using the SPH method and investigated the effects of presence of horizontal deck on wave kinematics. Le Touzé et al. [2010] employed SPH method for reproduction of green water phenomenon (flooding of a ship's deck) in two different scenarios. The first scenario corresponded to the interaction between a vessel and undulating travelling waves. The second scenario was related to the transient flooding behavior immediately after a side collision between two vessels.

Marrone et al. [2011] studied the wave pattern generated by high speed slender ships with sharp stems based on $2 \mathrm{D}+\mathrm{t}$ approach. According to $2 \mathrm{D}+\mathrm{t}$ method, the longitudinal gradients of flow quantities of interest were assumed to be small compared with vertical and transverse gradients. Accordingly, the steady 3D problem was simplified, leading to equations mathematically equivalent to those governing the unsteady $2 \mathrm{D}$ free-surface flow generated by a deformable body in the vertical plane transverse to the ship.

Marrone et al. [2012] carried out a precise analysis on 3D wave pattern generated by a ship in stationary forward motion with specific attention to the bow breaking wave using a 3D parallel SPH solver. Gao et al. [2012] applied a 2D SPH model for simulation of regular 
wave slamming on an open-piled structure. The model benefitted from kernel normalization (Corrected Smoothed Particle Method) and Riemann solver to discretize the continuity and Navier-Stokes equations. Barreiro et al. [2013] studied nonlinear behavior of fluid flow interacting with a rigid structure by using WCSPH (DualSPHysics code). The results of simulations were verified with respect to analytical solutions and experimental data. The model was used for simulation of complex wave-structure interaction, by reproducing a case study in real scale.

Altomare et al. [2014] performed 3D SPH-based simulations by using DualSPHysics code on run-up and wave interaction with a rubble mound breakwater. The response of armoured structure due to periodic wave was studied. The reproduced run-up heights were validated with respect to empirical solutions and experimental data. Altomare et al. [2015] implemented SPH (DualSPHysics) for simulation of wave action on coastal structures including vertical quays and storm return walls. The results of the simulation of regular and random wave interaction with vertical quay walls and storm return walls were verified with respect to semi-empirical solutions and experimental data. Wei et al. [2015] studied the impact of a tsunami bore on simplified bridge piers using SPH method on GPU. The effects of the shape and orientation of bridge pier with respect to free surface were carefully taken into account. The lea wake unsteady flow regimes and hydrodynamic force were investigated. Gong et al. [2016] proposed a two-phase liquid-gas WCSPH method for fluid-structure interactions with an improved solid boundary treatment for the considered rigid structures. Zheng et al. [2017b] presented an improved solid boundary treatment of ISPH by using a Simplified Finite Difference Interpolation (SFDI) scheme for the 2D wave-structure interaction problems. 
Lind et al. [2015] investigated the impact of a rigid horizontal plate onto a wave crest as well as a flat water surface by using a two-phase Incompressible-Compressible SPH (ICSPH) method where a projection-based ISPH for the liquid phase was coupled with a WCSPH for the gas phase. The coupling was made based on the imposition of kinematics from water phase on the air phase and imposition of pressure from the air phase on the water phase at the interface. The results obtained from numerical simulations were compared with experimental measurements. From the obtained results, it was concluded that air cushioning is of significant effect in case of impact on a flat water surface and this effect is reduced in case of waves, as the ratio of wave height to wave length increases.

Marrone et al. [2017] employed SPH for reproduction of the impact of smooth and corrugated panels on the water surface. Experimental studies on wet drop tests for both flat and corrugated panels were carried out and variations of pressures during the impact were investigated at several reference points along the panel surface. The model was first applied to the simulation of water entry test of a flat panel with a deadrise angle of $4^{\circ}$. Then, the slamming of Mark III type panel (a corrugated insulation panel), usually utilized in LNG tanks, was reproduced.

Fig. 2(a) shows the schematic side and bottom views of the modeled Mark-III insulation panel along with the position of pressure probes, for instance P53. Three sets of SPH-based simulations were conducted by Marrone et al. [2017] including 2D simulation with a single-phase model, 3D simulation with a single-phase model and 2D simulation with a two-phase model. Fig. 2(b) illustrates the snapshots corresponding to water impact of Mark III type corrugated insulation panel at $t=0.025 \mathrm{~s}$. The presented snapshots portray the robustness of SPH for detailed modeling of complex ocean engineering problems. Fig. 2(c) 
presents the results corresponding to the time history of pressure at probe P53 (close to the panel edge). From the presented figure, the most accurate results correspond to the two-phase SPH model, even if for P53, air cushioning is not expected to be essential.

Aristodemo et al. [2017] carried out numerical and experimental studies related to impacts of solitary waves on a submerged horizontal circular cylinder. The numerical study was performed by using a diffusive WCSPH. The acoustic waves produced within the numerical simulation of pressure field were filtered through the application of Wavelet Transform. The hydrodynamic coefficients in the Morison and transverse semi-empirical equations were calibrated based on the consistency in between experimental and numerical forces and kinematics at the cylinder.

Lind et al. [2017] conducted a study on the capabilities of multiphase Incompressible-Compressible SPH (ICSPH) [Lind et al., 2015] in estimation of the impact pressures due to the focused wave slamming on a fixed horizontal deck. The numerical results were verified against experimental data. Experiments were carried out in a flume of $17 \mathrm{~m}$ length, $1.3 \mathrm{~m}$ width and $1.0 \mathrm{~m}$ height. The study was limited to single focused wave interaction with the deck.

Fig. 3(a) illustrates a schematic sketch of the experimental conditions related to the deck along with the positions of pressure transducers (P1-P6) in the study by Lind et al. [2017]. The experimental conditions were adjusted as close as possible to a two-dimensional case, providing a consistent setup with numerical simulations. The computational domain is also portrayed in Fig. 3(a). The numerical simulation was initialized at $0.5 \mathrm{~s}$ before the focal time $t_{f}=21.36 \mathrm{~s}$ with the wave profile for the water phase provided by the linear theory. The boundaries of the water domain $\delta \Omega_{w}$ were set 
based on the predefined analytical pressure boundary conditions obtained from the linear wave theory, all through the domain, except at the intersection $\delta \Omega_{w} \cap \delta \Omega_{a}$, where the water boundary pressure was determined by the air phase (Fig. 3a). A buffer zone was designated at the outer region of the air phase at which air velocity was reduced using an exponential damping function. The dimensions of both the water and air phases, $\delta \Omega_{w}$ and $\delta \Omega_{a}$ respectively, varied in time, but initial setup was as $\delta \Omega_{w}=[7.5,8.5] \mathrm{m} \times[0.3,0.5+\eta] \mathrm{m}$ and $\delta \Omega_{a}=[7.6,8.4] \mathrm{m} \times[0.5+\eta, 0.75] \mathrm{m}$ where $\eta$ denotes the wave elevation at initial time $t_{0}=t_{f}=0.5 \mathrm{~s}$.

Fig. 3(b) and (c) depict the particles distribution as well as pressure contours obtained from Lind et al.'s ISPH-based simulations without and with considering the air phase, respectively, at $t=21.039$ s. From Fig. 3(c), air cushion has undergone a rapid collapse, due to the continued increase in ambient fluid pressure field from the progressing wave. This collapse is followed by a nearly horizontal slam of the water surface. On the other hand, Fig. 3(b) demonstrates progression of a contact line that continues to run smoothly along the deck underside with no significant slam event evident. Fig. 3(d) presents the pressure time history at P2 (Fig. 3a) in comparison with the experiment. It should be mentioned that the experimental results presented in this figure are not filtered. From Fig. 3d), the ICSPH has performed well in reproducing the envelope of the experimental time series, including the duration of the impact.

Bouscasse et al. [2017] carried out SPH-based simulations on fluid flow impacting a cylinder at or close to the free surface with Reynolds number of 180. The vortex formation patterns were studied for Froude numbers in between 0.3 and 2.0, based on the diameter, and for submergence-diameter ratios in between -0.5 and 2.5. Ferrand et al. [2017] 
presented an extension to the unified semi-analytical boundary conditions for SPH with Riemann solver for setting the unsteady open boundaries with complex shapes in confined and free-surface flows. The method was validated in simulation of water waves generation and free outlets.

\subsubsection{Fluid flow interactions with deformable structures}

The computational methods developed for simulation of fluid flow interactions with deformable elastic structures in the context of particle methods can be classified into two major categories as: i) FSI solvers developed by coupling the particle methods with other computational methods (either analytical or numerical) and ii) integrated fully-Lagrangian particle-based (meshfree) FSI solvers. As for the first category, i.e. partially particle-based FSI solvers, several efforts have been made for developing coupled solvers that concurrently benefit from appropriate Lagrangian formulation of particle-based methods along with taking advantage of straightforward and well-developed configurations of other computational frameworks. For example, Liang et al. [2017] employed Incompressible SPH method to investigate the impact of solitary waves on movable seawalls, represented as spring-controlled objects.

One of the most common strategies for computational FSI has been conducted by coupling the particle-based methods and Finite Element Method (FEM). In this regard, Fourey et al. [2010] coupled Weakly Compressible SPH (WCSPH) with FEM in simulation of violent interactions due to hydroelastic slamming. Fourey et al. [2017] made a comprehensive study on convergence and robustness of SPH-FEM coupling and presented an efficient coupled method for violent FSI modeling. 
Integrated particle-based FSI solvers, i.e. fully-Lagrangian meshfree coupled FSI solvers, are advantageous from different perspectives. First, an integrated fully-Lagrangian meshfree solver can potentially lead to precise and consistent imposition of fluid-structure interface boundary conditions. In addition, an integrated framework is advantageous from the viewpoint of adaptivity (multi-resolution modeling) and applicability. Also, in view of prominent capabilities of particle methods for both fluid and structural dynamics, e.g. fracture mechanics, an integrated FSI solver can be effectively further developed. As for integrated particle-based FSI solvers, Antoci et al. [2007] developed an integrated SPH-based coupled model for interaction between inviscid fluid flows with deformable structures. Oger et al. [2010] coupled WCSPH method with a hypoelastic SPH-based structure model in simulation of several test cases associated with hydroelastic slamming.

In the context of projection-based particle methods, Rafiee and Thiagarajan [2009] developed a fully-Lagrangian meshfree FSI solver by coupling a simplified projection-based incompressible SPH model with a hypoelastic SPH-based structure model. The term simplified is highlighted here, as the PPE in the work of Rafiee and Thiagarajan [2009] was solved in an explicit manner through an assumption of negligible pressure time variations. Hwang et al. [2014] developed an enhanced fully-Lagrangian FSI solver based on coupling between projection-based MPS method and a linear elastic structure model. The structure model was based on conservation equations of linear and angular momenta, for an isotropic linear elastic solid. The equation for conservation of linear momentum is described as: 


$$
\begin{gathered}
\left(\frac{D \boldsymbol{u}}{D t}\right)_{S}=\frac{1}{\rho_{S}} \nabla \cdot \boldsymbol{\sigma}_{S}+\boldsymbol{F}_{F t o S}+\boldsymbol{g} \\
\boldsymbol{\sigma}_{S}=\lambda_{S} \operatorname{tr}\left(\boldsymbol{\varepsilon}_{S}\right) \boldsymbol{I}+2 \mu_{S} \boldsymbol{\varepsilon}_{S}
\end{gathered}
$$

where, $\boldsymbol{u}$ represents velocity vector, $\rho_{S}$ stands for density of structure particle, $\sigma_{S}$ denotes the stress tensor of structure particle, $\boldsymbol{\varepsilon}$ is the strain tensor, $\boldsymbol{F}_{F \text { to } S}$ corresponds to the interaction force acting on interface from fluid $(F)$ to structure $(S)$ particles and $\boldsymbol{I}$ symbolizes the unit tensor. In Eq. (8), $\lambda_{S}$ and $\mu_{S}$ are Lame's constants, i.e. mechanical properties of the material, calculated from Young's modulus, $E_{S}$, and the Poisson's ratio, $v_{S}$, as follows:

$$
\begin{aligned}
& \lambda_{S}=\frac{E_{S} v_{S}}{\left(1+v_{S}\right)\left(1-2 v_{S}\right)} \\
& \mu_{S}=\frac{E_{S}}{2\left(1+v_{S}\right)}
\end{aligned}
$$

Conservation of angular momentum is involved in the solution process according to Eq. (10).

$$
\frac{D}{D t}(I \boldsymbol{\omega})_{S}=\frac{D}{D t}(\boldsymbol{r} \times m \boldsymbol{u})_{S}
$$

where $I, \omega$ and $m$ represent moment of inertia, angular velocity vector and mass of particle, respectively. 
Recently, Khayyer et al. [2017e] and [2017d] have presented some results of newly developed FSI solvers by coupling an enhanced projection-based Incompressible SPH fluid model with a SPH-based structure model as well as an enhanced MPS fluid model with a MPS-based structure model, referred to as Enhanced ISPH-SPH and Enhanced MPS-MPS, respectively. An important fact in development of these newly developed FSI solvers corresponds to exact satisfaction of fluid-structure interface boundary conditions including continuity of normal stress. Fig. 4 shows some qualitative simulation results of hydrodynamic slamming tests on Solid Glass (SG) fiber single skin panel [Allen, 2013] by Enhanced ISPH-SPH and MPS-MPS FSI solvers. In the experiment, the panels of $1.03 \mathrm{~m}$ length and $0.6 \mathrm{~m}$ width were being carried on a rig capable of driving either with constant or variable velocities in the range of deadrise angles from $0^{\circ}$ up to $40^{\circ}$. The extremities of the panel were simply supported by a fixture frame, setting an unsupported area of $0.99 \mathrm{~m}$ by $0.485 \mathrm{~m}$ between simply supported edges of the test fixture. The lower supported edge was representative of keel while the upper as chine. The $9.5 \mathrm{~mm}$ thick SG panel was of 1.52 $\mathrm{kNm}$ bending stiffness and $4.4 \mathrm{E}+04 \mathrm{kN} / \mathrm{m}$ shear stiffness. With respect to the experimental restraining conditions, for $2 \mathrm{D}$ simulations the panel was restrained at two points leading to a free span of $0.485 \mathrm{~m}$, representing the inner edges of the fixture frame, as shown in Fig. 4(a). The initial particle spacing in simulations was set as $0.0019 \mathrm{~m}$.

Fig. 4(b-e) demonstrates a set of snapshots corresponding to the test case of SG slamming at water surface with a velocity of $4 \mathrm{~m} / \mathrm{s}(v=4 \mathrm{~m} / \mathrm{s}, v$ is the vertical component of velocity) by Enhanced MPS-MPS and ISPH-SPH FSI solvers at $t=20 \mathrm{~ms}$ and $t=24 \mathrm{ms,}$ respectively. From the presented figure, high-level performance of FSI solvers in reproduction of stable/smooth stress/pressure fields is evident. The results were also 
compared against experimental data in terms of time histories of structural deflection and hydrodynamic pressure field at specific measuring points.

\subsection{Exclusive applications for coastal/ocean engineering problems}

Particle methods have been directly and exclusively applied to solve several important coastal/ocean engineering problems. Indeed, the fully-Lagrangian meshfree features of particle methods would make them excellent candidates to solve problems that are not easy to be precisely/flexibly targeted by conventional grid-based methods. Examples include, simulation of floating bodies (especially those under extreme sea states), aerated wave breaking, violent wave impacts, surf/swash zone hydrodynamics and sediment transport. In the following subsections, several exclusive applications of particle methods for coastal/ocean engineering problems are reviewed. These exclusive applications are divided into three categories of floating bodies, coastal hydrodynamics and coastal sediment transport.

\subsubsection{Floating bodies}

One of the most interesting applications of particle methods for coastal/ocean engineering corresponds to the modeling of floating objects including those moored to the sea bottom. In this regard, particle methods are especially well suited for violent fluid flows interacting with floating bodies of complex geometries, due to their meshfree Lagrangian features, as highlighted by Liu and Liu [2015]. Gómez-Gesteira et al. [2012a] described the SPH-based formulations used in SPHysics along with enhancements in terms of density filtering, Arbitrary Lagrange-Euler (ALE) schemes and Riemann solvers. Also boundary conditions 
employed in SPHysics capable of handling floating objects in fluid-structure interactions were introduced. They presented the results of simulation of wave evolution by conservative and non-conservative Riemann solvers. Bouscasse et al. [2013] applied a Weakly Compressible SPH (WCSPH) solver for simulation of nonlinear interaction of water waves and floating bodies. Ghost-fluid technique was used for imposition of no-slip boundary condition on the solid surface. They developed an explicit synchronous algorithm for coupling the fluid and rigid bodies.

Bilotta et al. [2014] developed a SPH-based model for simulation of 3D dam-break on a natural topography with floating objects. The boundaries were set according to unified semi-analytical boundary model of Ferrand et al. [2012] based on a re-normalizing factor for enforcing the boundary terms. This factor depends on the local shape of a wall and on the position of a particle relative to the wall. The 3D SPH simulations were conducted by CUDA-enabled GPUs, leading to high computing performance.

Ren et al. [2015] studied nonlinear interactions between waves and floating bodies by WCSPH method. They proposed an improved treatment algorithm for moving boundary of the floating body based on the Dynamic Boundary Particles (DBPs). The DBPs follow the same equations of continuity and state as the fluid particles, but not the same momentum equation. The forces exerted on the floating body boundary particles were estimated using the volume integration of the stress tensors. Ren et al. [2017] simulated the nonlinear interactions between waves and moored floating breakwater by using WCSPH. Solid boundaries were treated as two layers of DBPs. The mooring line system was modeled as a light spring model and the gravity effect and volume of the mooring lines were neglected. 
The interactions between waves and structures were modeled by setting a series of SPH particles on the boundary of the structure.

Lind et al. [2016a] employed ISPH for evaluation of the hydrodynamic loads due to breaking/non-breaking waves, within inertia-dominated ranges of waves. The hydrodynamic wave-induced forces on the bodies of relatively small diameter (with respect to wave length) were calculated based on Froude-Krylov force, along with an additional term associated with added mass. According to the results of their study, breaking wave force magnification was consistent with the experiment in case of fixed vertical column exposed to regular waves on a small slope. For the case of focused waves, the peak forces due to initial interaction were reported to be in good agreement with the experiment. Fig. 5(a) and (b) demonstrate the formation of plunging wave breaker of focused wave with jet impacting the cylinder. The wave maker input was validated and good agreement was obtained in reproducing the wave elevation in front of the cylinder as it can be observed in Fig. 5(c) (especially before and at the instant of plunging jet impact, $t \approx 11.8 \mathrm{~s}$ at Fig. 5a). In addition, the estimated forces by using the concept of Froude-Krylov were in relatively good agreement with those measured in the experiment (Fig. 5d). It was concluded that, despite the relatively high velocities of the plunging jet (see Fig. $\mathbf{5 b}$ ), in this case, taking just undisturbed flow field into consideration does make sense and results in a reasonable agreement in terms of the total loading on the cylinder. In the case of taut moored hemispherical buoy exposed to steep focused waves, the loads and motion without snatching were likely to be approximately independent of added mass coefficient. 
Crespo et al. [2017] applied a well-known open source SPH-based code, namely, DualSPHysics, for simulation of interaction between sea waves and an Oscillating Water Column device (OWC). The effect of mooring systems on a floating offshore OWC was studied by using WCSPH. The simulations were conducted in single-phase. The setup of the computational domain is shown in Fig. 6. The configuration and dimensions of the OWC was set based on computational purposes rather than realistic OWC. The channel was of $12.8 \mathrm{~m}$ width and $190 \mathrm{~m}$ length, with a $57 \mathrm{~m}$ distance in between piston and the OWC. The maximum depth was $10 \mathrm{~m}$ with a flat region of $80 \mathrm{~m}$ connected to a beach of $8^{\circ}$ slope. The floating OWC device itself was $3 \mathrm{~m}$ tall and $2.2 \mathrm{~m}$ wide with a $1 \mathrm{~m}$ tall opening. The device was supported by a squared floating structure. The whole system was exposed to open sea extreme wave conditions and was moored to the sea bottom by four lines with a total length of $14 \mathrm{~m}$ and a wet volumetric density of $7850 \mathrm{~kg} / \mathrm{m}^{3}$. The initial particle spacing was set as $0.15 \mathrm{~m}$, leading to a total number of 3,700,000 particles. Regular waves ( $H=1.8 \mathrm{~m}, T=9.0 \mathrm{~s}, d=10 \mathrm{~m}$ with $L=81 \mathrm{~m})$ were reproduced as preliminary results of free-surface oscillations inside an offshore floating OWC in an open ocean. A 3D numerical tank was designed with a passive wave absorption in form of dissipative beach at the end of the tank and an active wave absorption system (AWAS) to absorb any reflected wave energy from the impact with the external front wall of the floating OWC. Open periodic boundaries were used in the direction perpendicular to the incoming wave direction. Fig. 6(a-f) demonstrates snapshots corresponding to the velocity field at different instants of the simulation. As it can be seen, by modeling only regular non-breaking waves with the use of an active wave absorption system, a regular pattern of surface oscillations could be obtained. 
Canelas et al. [2017a] proposed an integrated framework for modeling fluid-structure-structure coupled systems, by implementing the Project Chrono library in the DualSPHysics code. They applied the coupled method into several cases as fully jointed WaveStar Wave Energy Converter (WEC) with a non-linear power take-off system, a tidal turbine with two relative degrees of freedom and a water mill allowed for rotation due to the impact of a water jet. The DVI implementation of Project Chrono provided precise Coulomb friction modeling for sticking, rolling and sliding contacts. Fig. 7 shows a WaveStar machine idealized as a WEC driving a set of half-submerged buoys. Under normal operating conditions, a trivial linear analysis model can be used to recover the machine response, but CFD or experimental set-ups were required to analyze extreme conditions. With setting the regular wave maker, $2^{\text {nd }}$ order waves of the same amplitude as the height of the buoys and a wavelength equal to their distance were generated, causing breaking at the buoy locus.

By introducing a Rigid Module and Flexible Connector (RMFC) multi-body system within a multi-phase SPH-based method, Shi et al. [2018] reproduced the failure process of a flexible floating oil boom. Simulations of the dynamics of a flexible floating boom containing industrial gear oil were conducted under the action of combined waves and currents. Good agreements were achieved in between SPH simulation results and the experimental data in terms of the ambient wave-current field, hydrodynamic responses of the floating body and evolution process of the oil slick for the flexible boom. Fig. 8 portrays a set of snapshots illustrating different stages of oil slick propagation due to variation of current velocity. The figure depicts the capability of SPH in reproducing fluid current interaction with a flexible floating oil boom. 
The dimensions of the boom in the experiment conducted by Shi et al. [2018], presented in Fig. 8, were set as; floater diameter equal to $0.1 \mathrm{~m}$, skirt length as $0.1 \mathrm{~m}$ and skirt thickness as $0.005 \mathrm{~m}$. The skirt of the flexible boom comprised of 4 modules connected by freely-rotating connectors. The computational domain for SPH-based simulations was set as $10.0 \mathrm{~m}$ length with the floating boom placed in the middle of the flume. A $0.02 \mathrm{~m}$ thick slick of industrial gear lubricant CKC680 with molecular viscosity and reference density being $1625 \mathrm{~mm}^{2} / \mathrm{s}$ and $890 \mathrm{~kg} / \mathrm{m}^{3}$ was spread on the upper layer of water surface within a length $1 \mathrm{~m}$ at the upstream side of bloom. The initial particle spacing was set as $0.005 \mathrm{~m}$ for all the particles.

\subsubsection{Coastal hydrodynamics}

With respect to crucial properties of coastal/ocean hydrodynamics characterized by violent/complex free surface flows, e.g. in wave breaking, overtopping, run-up, wave impact, green water, etc., Lagrangian meshfree particle methods have been proven to be excellent computational candidates [Violeau and Rogers, 2016; Gotoh and Okayasu, 2017]. Accordingly, many efforts have been dedicated to development of particle-based computational methods for application in crucial problems associated with coastal hydrodynamics.

Dalrymple and Rogers [2006] presented the capabilities of SPH method in reproduction of breaking waves on beaches in two and three dimensions, green water overtopping on decks, and wave-structure interaction. The method was enhanced with Shephard filtering scheme, new time stepping and Sub-Particle-Scale turbulence model [SPS; Gotoh et al., 2001]. Crespo et al. [2008] implemented SPH method for simulation of wave propagation 
toward the coast and for analyzing the nature of overtopping on coastal structures under storm condition. Li et al. [2012] investigated the propagation of solitary wave in shallow water by using WCSPH method. The model was verified for the case of incident solitary wave propagating on various bed configurations. The fission processes of both non-breaking and breaking solitary waves were studied. Gomez-Gesteira et al. [2012b] examined the efficiency of SPHysics by reproducing creation of waves by landslides, dam-break propagation over wet beds and wave-structure interaction.

Farahani and Dalrymple [2014] studied the turbulent vortical structures under broken solitary waves by using the SPH method. They investigated the generation mechanisms and geometric configurations of coherent structures under broken solitary waves. Marrone et al. [2015] investigated the energy dissipation involved in liquid impacts in both of the frameworks of the weakly compressible SPH (WCSPH) and incompressible models (Level-Set Finite Volume Method). They carried out their study on the impact of two water jets in $1 \mathrm{D}$ and 2D framework, representing the prevalent phenomena during the evolution of plunging wave breaker, being responsible for a considerable amount of the energy loss. Altomare et al. [2015] presented a hybrid SWASH (Simulating WAve till SHore)-SPH model for real coastal applications, and more specifically, for interaction of sea waves with coastal structures. In that study, a one-way coupling in between SWASH wave model and DualSPHysics was carried out with a hybridization point where the information of the generated waves obtained by SWASH were passed to DualSPHysics. Waves in DualSPHysics were generated by means of a moving boundary whose displacement in time was reconstructed using the velocities provided by SWASH. 
Wen et al. [2016] developed a WCSPH-based method in closure with SPS turbulence model (Gotoh et al. [2001]) for 3D simulation of wave interaction with coastal structures. The model was applied to simulate wave interactions with a vertical breakwater and a vertical cylinder. The developed SPH code enabled the construction of an appropriate numerical wave basin, allowing simulation of larger wave fields than those considered in commonly developed SPH models.

Makris et al. [2016] performed a numerical study on the propagation and breaking of shore-normal, nonlinear and regular waves. The 2D kinematics and dynamics of wave induced regimes in a surf-zone were reproduced by using SPHysics. Altomare et al. [2017] performed comprehensive simulations on wave generation and absorption for second-order long-crested monochromatic and random waves by using WCSPH-based DualSPHysics code. A piston-type wave maker was considered and second-order regular and random waves were generated along with passive and active wave absorption systems. Passive absorption was produced by using a damping system to prevent wave reflection from fixed boundaries of the numerical flume. The active wave absorption was designated to prevent spurious reflection from the wave maker. These implementations were validated with theoretical solutions and experimental results, in terms of water surface elevation, wave orbital velocities, wave forces and capacity for damping the re-reflection inside the fluid domain.

Zhang et al. [2017] presented a low-dissipation WCSPH method for modeling violent free surface flows by modifying the Riemann solver, ensuring minimized numerical dissipation for expansion or compression waves. In addition, wall boundary conditions based on the one-sided Riemann solver were imposed capable of handling violent 
breaking-wave impacts. Yeganeh-Bakhtiary et al. [2017] conducted a study on the hydrodynamics of standing waves interacting with a caisson breakwater by a 2D WCSPH model. The effects of wall steepness on the wave hydrodynamics were investigated by consideration of the patterns of steady streaming for both fully and partially standing waves.

In the context of projection based methods, Akbari [2017] carried out simulations on wave overtopping by using SPH method for various coastal structures. The viscosity of surface particles was modified based on the concept of surface viscosity [Xu, 2010]. The value of the introduced surface viscosity depended on flow velocity, velocity gradient, modeling condition and shape of the utilized kernel. The proposed model was verified in simulation of different cases of dam break, solitary wave breaking and wave overtopping over vertical and sloping seawalls.

\subsubsection{Coastal sediment transport}

Sediment transport regimes caused by coastal hydrodynamics have been always as important challenges in coastal engineering. Thus, several studies have targeted simulation of these important phenomena within Eulerian, Lagrangian or hybrid frameworks. In this regard, particle methods can be appropriate candidates for reproducing problems associated with sediment transport, specially when they are carefully and consistently coupled with other Lagrangian methods, e.g. DEM [Discrete Element Method; Cundall and Strack., 1979].

As for integrated two-phase particle-based methods, Gotoh and Sakai [2006] presented a review on the schemes developed for particle methods with applications for coastal 
sediment transport. Ikari et al. [2010] developed an erosion model by incorporation of an elasto-plastic constitutive equation in MPS-based model for time-dependent analysis of large deformations of soil, due to wave induced erosion in sea cliffs. The mentioned model was further enhanced by utilizing sub-particle-scale suspended sediment load model together with advection-diffusion equation by Ikari et al. [2015].

Ran et al. [2015] developed an ISPH-based two-phase solid-liquid model in closure with SPS turbulence model to simulate the scour process due to dam break flows. The erosion model was set according to the formulations proposed by Ikari et al. [2010], based on the effective pick-up flow velocity concept. Wang et al. [2016] presented an ISPH Simulation of scour behind seawall due to continuous tsunami overflow. The concepts of numerical turbid water particle and clear water particle were proposed to deal with the sediment-entrained flow. The initiation of sediment particle movement was determined by consideration of the concept of critical shear stress.

Fourtakas and Rogers [2016] employed a two-phase solid-liquid model based on $\delta$-SPH for simulation of global erosion processes including yielding, shear and suspension layer. The Herschel-Bulkley-Papanastasiou (HBP) model was employed to reproduce the rheological characteristics of the yielded sediment layer as a non-Newtonian fluid along with a Drucker-Prager yield criterion at the fluid-soil interface. The model was verified in a 3D dam break over a non-cohesive erodible bed simulation.

Shi et al. [2017] proposed a two-phase solid-liquid SPH-based model to reproduce the sediment motion in free surface flows. The water and the sediment were modeled as two miscible fluids and the multi-fluid system was discretized by a single set of SPH particles. The Macdonald-Tait equation of state was proposed for defining the pressure at the 
sediment-water mixture. Ghaïtanellis et al. [2018] coupled a multi-fluid formulation of SPH with an elastic-viscoelastic constitutive equation to model non-cohesive sediment transport. A shear-thinning non-Newtonian fluid model was set for the yielded sediment while Drucker-Prager's criterion was assigned as the yielding criterion. The model was verified in a 2D soil collapse test case and a dam break over mobile bed.

Regarding coupling the particle methods developed for continuum mechanics and DEM, Sun et al. [2013] proposed a coupled DEM-SPH method, with conservative momentum transformations on the basis of a variational approach. Canelas et al. [2016] proposed a unified discretization of rigid solids and fluids with resolved representation of solid-fluid phases. Based on coupling between SPH and a variation of DEM (Distributed Contact DEM or DCDEM), a coupled SPH-DCDEM method was developed. The SPH-DCDEM discretization, allows for application of contact theories to arbitrary geometries of rigid solids with exact transfer of momentum. Canelas et al. [2017b] presented impressive and highly-resolved simulations of stony debris flows by using their SPH-DCDEM method.

Harada et al. [2017] developed a DEM-MPS coupled method for reproduction of swash beach sediment transport processes and highlighted the effect of infiltration/exfiltration in sediment transport regimes corresponding to a gravel beach. Prominent capabilities of coupled DEM-MPS for modeling problems with complex moving boundaries, such as the water surface of the breaking wave and the surface of the bottom movable bed within swash zone were described. Harada et al. [2018] investigated the formation/deformation processes of step series on the riverbeds in mountain areas by using coupled DEM-MPS method. They showed the capabilities of DEM-MPS coupled method as a promising tool for investigating morphological dynamics of the step series. The exfiltration process of dye 
was reproduced well by DEM-MPS that validated the accuracy of this coupling approach for reproduction of seepage flow.

\subsection{Enhancement of computational efficiency}

One of the most challenging issues related to particle methods corresponds to the required high computational costs, that may downgrade their efficiency in terms of applications for practical/critical engineering problems. Therefore, within recent years, great efforts have been devoted to the development of parallel computing techniques, especially GPGPU (General-Purpose computing on Graphics Processing Unit), for improvement of computational efficiency.

In the framework of weakly compressible SPH, thanks to its explicit algorithm, implementation of parallel computing technique is relatively straightforward and therefore several studies have been dedicated to this subject, so far. In this regard, Hérault et al. [2010] implemented SPH on GPU for free surface flows by using the Compute Unified Device Architecture (CUDA), resulting in considerable speed-ups (by up to two orders of magnitude faster than the equivalent CPU-based simulation). Crespo et al. [2011] presented SPH-based code of DualSPHysics either based on GPU or CPU implementation. Application of GPU resulted in remarkable speedups in computations (by up to two orders of magnitude). They utilized single GPU-card instead of single-core CPU for simulations, dealing with computational domains with numbers of particles beyond one million. DualSPHysics GPU portion was comprehensively validated in a variety of problems associated with coastal/ocean engineering, e.g. by Altomare et al. [2014] and [2017], Domínguez et al. [2013], Barreiro et al. [2013], Fourtakas and Rogers [2016], Crespo et al. 
[2015] and [2017]. Oger et al. [2016a] highlighted various key issues corresponding to massive parallelization of explicit particle methods on MPI (Message Passing Interface; process parallel computing)-based distributed memory parallelization.

In the context of projection-based particle methods and through a pioneering study, Hori et al. [2011] implemented MPS method on GPU. In their study, the speedup was limited by only one order of magnitude, mainly due to iterative procedure of the solution of Poisson Pressure Equation (PPE). The same order of speedup was achieved in other GPU implementations of projection-based particle methods, e.g. GPU implementations of MPS [Kakuda et al. 2013] and ISPH [Qiu, 2014].

\section{Future perspectives}

In general, particle methods have shown a great potential for presenting substantial contributions to significant problems associated with coastal/ocean engineering, due to their meshfree Lagrangian formulations. However, in spite of prominent advancements achieved in enhancement of stability and accuracy of particle methods, further rigorous and comprehensive investigations must be conducted in order to develop these new generation computational methods into thoroughly reliable and robust design tools for practical engineering purposes.

In this regard, Smoothed Particle Hydrodynamics European Research Interest Community (SPHERIC) has identified a set of so-called Grand Challenges towards comprehensive development of particle methods including: convergence, consistency, stability, boundary conditions, adaptivity, coupling to other models and applicability to the industry. Although, particle-based methods have been subjected to substantial 
enhancements in terms of stability, accuracy and boundary conditions, there is still much to be done in regard to convergence and adaptivity [Violeau and Rogers, 2016].

Convergence, i.e. consistency of the discretized form of differential equation with corresponding continuous form, is one of the challenging aspects of particle methods which has to be scrupulously studied in future. In fact, due to the difficulties involved in achieving higher orders of convergence, researchers have mainly focused on enhancement of accuracy rather than convergence itself. Therefore, increasing the global order of convergence to the same order of general mesh-based methods, is still an ongoing development [Lind and Stansby, 2015; Shadloo et al., 2016].

Regarding stability enhancement, advancements of stabilization schemes such as the Particle Shifting [Lind et al., 2012], Optimized Particle Shifting [Khayyer et al., 2017a] or Dynamic Stabilization [Tsuruta et al., 2013] are to be undertaken, in order to guarantee the methods' stability for a wide range of applications. The probable adverse effects of stabilizing schemes in terms of conservation and convergence must be carefully and rigorously studied.

As for the accuracy aspect, despite significant improvements, the problem of unphysical pressure fluctuations remains to be not meticulously resolved. Further enhancements of accuracy are expected to be achieved, thanks to the profound and thorough studies that are being conducted accordingly. Further refinement of differential operator models, such as gradient and Laplacian operators, corresponding to the terms that directly appear in the considered governing equations, and applications of higher-order accurate numerical solution processes (e.g. higher-order projection methods) are instances of prospective 
advancements that are likely to be conducted for enhancement of accuracy of particle methods.

As for energy conservation properties of particle methods, special focus should be given to revision/derivation of formulations with respect to an energy-based framework. Energy conservation properties are to be considered as one of the most essential aspects for validation of newly developed schemes in all aspects of particle method applications, e.g. multi-phase flows, FSI simulations, coupled methods, boundary conditions and etc.

With respect to the application of particle methods into practical purposes related to coastal/ocean engineering, more attractive aspects of the development for industrial projects will be emphasized here as adaptivity, multi-phase/multi-scale simulations, Fluid Structure Interaction (FSI), advancement of coupling schemes and turbulence models. In this regard, one of the prospective features of interest for development of particle-based methods corresponds to the advancement of adaptive multi-resolution schemes, leading to significant improvements in computational efficiency. In future, substantial developments are expected for particle-based multi-resolution schemes, both in terms of efficiency and performance. Application of the advanced versions of multi-scale schemes will be accompanied by rigorous considerations regarding conservations of volume, momentum and energy.

As for the multiphase simulations, especially those characterized by large density ratios, the currently developed particle methods consider some numerical treatments, such as stabilizers or smoothing schemes. In future, more mathematically sound, physically rigorous schemes are to be developed for particle-based simulation of multiphase flows, with specific attention to detailed physics of the problem as well as conservation properties 
in terms of volume, momentum and energy. Particle methods are expected to be applied in simulation of complex multi-phase phenomena including phase transitions, for instance, that are currently beyond their capabilities.

As for Fluid-Structure Interactions (FSI) corresponding to deformable structures, further enhancements are to be achieved in particle-based structure models in terms of stability, accuracy and conservation properties. Prevalent instabilities in terms of stress state instability and rank deficiency are to be perfectly removed by incorporation of relevant consistent schemes (e.g. combined use of Lagrangian kernels for tensile instability and stress points for rank deficiency). Implementation of implicit solvers for particle-based structure models will potentially bring about further enhancements of stability as well as accuracy. Particle-based models are expected to be further developed for reliable simulation of complex/challenging problems characterized by material discontinuities, including fracture mechanics and dynamics of composite structures. More consistent coupling schemes are to be developed with rigorous considerations for exact satisfaction of fluid-structure interface boundary conditions. Multi-scale simulations are expected to gain more interest in practical particle-based FSI simulations, mainly for achievement of physically-consistent computationally-efficient simulations.

As for coupling of particle-based methods and other computational frameworks, regardless of the specific application (e.g. sediment transport, scouring, erosion, FSI), the most important issue is related to treatment of interfaces and more specifically, accurate implementation of boundary conditions at the (phase and/or computational) interfaces. In future, more advanced (physically-consistent, mathematically-sound and numerically-accurate) coupling schemes are to be developed, with gradual enhancement of 
conservation properties related to momentum, energy and volume [Canelas et al., 2016; Harada et al., 2017]. The coupled methods should be validated through more challenging problems, with inclusion of complex mutual interactions in between different phases. By coupling/merging particle methods with other computational frameworks, the resulting hybrid methods [e.g. Voronoi-SPH by Fernandez-Gutierrez et al., 2017; SPH-FEM by Fourtakas et al., 2017; Finite Volume-SPH by Marrone et al., 2016; ALE (Arbitrary Eulerian-Lagrangian)-ISPH by Lind and Stansby, 2016; ALE-LSMPS (Least Squares MPS) by $\mathrm{Hu}$ et al., 2017a; and ELI (Eulerian-Lagrangian Incompressible)-SPH by Fourtakas et al., 2018] will simultaneously take advantage of prominent privileges included in different frameworks, complementing the shortcomings of each framework.

In regard to turbulence modeling, up to now, several studies have incorporated different types of turbulence models in the context of both explicit particle methods [e.g. Issa et al., 2010] and the semi-implicit projection-based ones [e.g. Shao and Lo, 2003; Gotoh and Sakai, 2006; Leroy et al., 2015]. Research on proper modeling of turbulence by either time-averaged [e.g. Violeau and Issa, 2007] or spatially-averaged [e.g. Sub-Particle-Scale; Gotoh et al., 2001] turbulence models is continuously advancing [e.g. Mayrhofer et al., 2015].

In all cases, it is important to keep the developed numerical methods free of any numerical term with constants that require calibration. This important issue is also highlighted in the review paper by Violeau and Rogers [2016]. Indeed, prior to any practical application, rigorous verification of particle-based codes must be conducted by consideration of appropriate benchmark tests with exact analytical solutions in terms of 
reproduced velocity/pressure together with comprehensive investigations on conservation and convergence properties.

\section{Concluding remarks}

Recent advancements corresponding to particle-based computational methods, particularly for coastal/ocean engineering applications are discussed, in both frameworks of Weakly Compressible SPH (WCSPH) and projection-based particle methods, including Incompressible SPH (ISPH) and Moving Particle Semi-implicit (MPS) methods. Latest achievements related to particle methods and corresponding to enhancements of stability, accuracy, energy conservation, multi-phase, multi-physics and multi-scale simulations, Fluid-Structure Interactions (FSI), exclusive coastal/ocean engineering applications and computational efficiency are reviewed. The key issues and substantial challenges in each category are highlighted. Prospective features for further development of particle methods as reliable and robust computational methods for practical engineering purposes are discussed.

\section{Acknowledgments}

The authors would like to express their gratitude to Dr. Andrea Colagrossi and Dr. Salvatore Marrone at CNR-INSEAN (The Italian Ship Model Basin), Dr. Benedict Rogers and Dr. Steven Lind at the University of Manchester, Dr. Alejandro Crespo at Universidade de Vigo, Professor Shaowu Li and Mr. Yang Shi at Tianjin University for kindly providing figures related to their latest studies. 


\section{References}

Adami, S., Hu, X.Y. \& Adams, N.A. [2013] "A transport-velocity formulation for smoothed particle hydrodynamics," Journal of Computational Physics 241, 292-307.

Allen, T. [2013] "Mechanics of flexible composite hull panels subjected to water impacts," Ph.D. Thesis, University of Auckland.

Altomare, C., Crespo, A.J.C. Rogers, B.D., Domínguez, J.M., Gironella, X. \& Gómez-Gesteira, M. [2014] "Numerical modelling of armour block sea breakwater with smoothed particle hydrodynamics," Computers and Structures 130, 34-45.

Altomare, C., Crespo, A.J.C., Domínguez, J.M., Gómez-Gesteira, M., Suzuki, T. \& Verwaest, T. [2015] "Applicability of Smoothed Particle Hydrodynamics for estimation of sea wave impact on coastal structures," Coastal Engineering 96, 1-12.

Altomare, C., Domínguez, J.M., Crespo, A. J. C., Suzuki, T., Caceres, I. \& Gómez-Gesteira, M. [2015] "Hybridization of the Wave Propagation Model SWASH and the Meshfree Particle Method SPH for Real Coastal Applications," Coastal Engineering Journal 57 (4), 1550024.

Altomare, C., Domínguez, J.M., Crespo, A.J.C., González-Cao, J., Suzuki, T., Gómez-Gesteira, M. \& Troch, P. [2017] "Long-crested wave generation and absorption for SPH-based DualSPHysics model," Coastal Engineering 127, 37-54.

Akbari, H. [2017] "Simulation of wave overtopping using an improved SPH method," Coastal Engineering 126, 51-68.

Antoci, C., Gallati, M. \& Sibilla, S. [2007] "Numerical simulation of fluid-structure interaction by SPH," Computers and Structures 85(11), 879-890. 
Antuono, M, Colagrossi, A., Marrone, S. \& Molteni, D. [2010] "Free-surface flows solved by means of SPH schemes with numerical diffusive terms," Computer Physics Communications 181, 532-549.

Antuono, M., Colagrossi, A. \& Marrone, S. [2012] "Numerical diffusive terms in weakly-compressible SPH schemes," Computer Physics Communications 183, $2570-2580$.

Antuono, M., Marrone, S., Colagrossi, A. \& Bouscasse, B. [2015] "Energy balance in the $\delta$-SPH scheme," Computer Methods in Applied Mechanics and Engineering 289, $209-226$

Aristodemo, F., Tripepi, G., Meringolo, D.D. \& Veltri, P. [2017] "Solitary wave-induced forces on horizontal circular cylinders: Laboratory experiments and SPH simulations," Coastal Engineering 129, 17-35.

Barreiro, A., Crespo, A.J.C., Domínguez, J.M. \& Gómez-Gesteira, M. [2013] "Smoothed Particle Hydrodynamics for coastal engineering problems," Computers and Structures 120, 96-106.

Bilotta, G., Vorobyev A., Hérault A., Violeau D. \& Negro C.D. [2014] "SPH for the simulation of a dam-Break with floating objects, ” In: Russo G., Capasso V., Nicosia G., Romano V. (eds) Progress in Industrial Mathematics at ECMI 2014. ECMI 2014. Mathematics in Industry, vol 22. Springer, Cham.

Bouscasse, B., Colagrossi, A., Marrone, S. \& Antuono, M. [2013] "Nonlinear water wave interaction with floating bodies in SPH," Journal of Fluids and Structures 42, 112-129. 
Bouscasse, B., Colagrossi, A., Marrone, S. \& Souto-Iglesias, A. [2017] "SPH modelling of viscous flow past a circular cylinder interacting with a free surface," Computers and Fluids 146, 190-212.

Bui, H.H. \& Nguyen, G.D. [2017] “A coupled fluid-solid SPH approach to modelling flow through deformable porous media," International Journal of Solids and Structures 125, 244-264.

Canelas, R.B., Crespo, A.J.C., Domínguez, J.M., Ferreira, R.M.L. \& Gómez-Gesteira, M. [2016] "SPH-DCDEM model for arbitrary geometries in free surface solid-fluid flows," Computer Physics Communications 202, 131-140.

Canelas, R.B., Brito, M., Ferreira, R.M.L., Feal, O.G., Domínguez, J.M. \& Crespo, A.J.C. [2017a] "DualSPHysics, applications of a HPC multiphysics simulation framework," Proceedings of SPHERIC 2017, $12^{\text {th }}$ international SPHERIC workshop, Ourense, Spain, June $13-15$.

Canelas, R.B., Domínguez, J.M., Crespo, A.J.C., Gómez-Gesteira, M. \& Ferreira, R.M.L. [2017b] "Resolved simulation of a granular-fluid flow with a coupled SPH-DCDEM model," Journal of Hydraulic Engineering 143(9), 06017012.

Cercos-Pita, J.L., Dalrymple, R.A. \& Hérault, A. [2016] "Diffusive terms for the conservation of mass equation in SPH," Applied Mathematical Modelling 40, $8722-8736$.

Cercos-Pita, J.L., Antuono, M., Colagrossi, A. \& Souto-Iglesias, A. [2017] "SPH energy conservation for fluid-solid interactions," Computer Methods in Applied Mechanics and Engineering 317, 771-791. 
Chorin, A.J. [1968] "Numerical solution of the Navier-Stokes equations," Mathematics of Computations 22, 745-762.

Coetzee, C.J. [2017] "Review: Calibration of the discrete element method," Powder Technology 310, 104-142.

Colagrossi, A. \& Landrini, M. [2003] "Numerical simulation of interfacial flows by smoothed particle hydrodynamics," Journal of Computational Physics 191(2), 448-475.

Colagrossi, A., Antuono, M., Souto-Iglesias, A. \& Le Touzé, D. [2011] “Theoretical analysis and numerical verification of the consistency of viscous smoothed-particle-hydrodynamics formulations in simulating free-surface flows," Physical Review E 84(2), 0.39901.

Colagrossi, A., Marrone, S., Bouscasse, B. \& Broglia, R. [2015] "Numerical Simulations of the Flow Past Surface-Piercing Objects," International Journal of Offshore and Polar Engineering 25(1), 13-18.

Crespo, A.J.C., Gómez-Gesteira, M., Carracedo, P. \& Dalrymple, R.A. [2008] "Hybridation of generation propagation models and SPH model to study severe sea states in Galician Coast," Journal of Marine Systems 72, 135-144.

Crespo, A.J.C., Domínguez, J.M., Barreiro, A., Gómez-Gesteira, M. \& Rogers, B.D. [2011] "GPUs, a new tool of acceleration in CFD: Efficiency and reliability on smoothed particle hydrodynamics methods," PLOS ONE 6, e20685.

Crespo, A.J.C., Domínguez, J.M., Rogers, B.D., Gómez-Gesteira, M., Longshawb, S., Canelas, R., Vacondio, R., Barreiro, A. \& García-Feal, O. [2015] "DualSPHysics: 
Open-source parallel CFD solver based on Smoothed Particle Hydrodynamics (SPH),” Computer Physics Communications 187, 204-216.

Crespo, A.J.C., Altomare, C., Domínguez, J.M., González-Cao, J. \& Gómez-Gesteira, M. [2017] "Towards simulating floating offshore oscillating water column converters with Smoothed Particle Hydrodynamics," Coastal Engineering 126, 11-26.

Cummins, S.J. \& Rudman, M. [1999] “An SPH projection method," Journal of Computational Physics 152, 584-607.

Cundall, P.A, \& Strack, O.D.L. [1979] "A discrete numerical model for granular assemblies," Geotechnique 29, 47-65.

Dalrymple, R.A. \& Rogers, B.D. [2006] "Numerical modeling of water waves with the SPH method," Coastal Engineering 53(2), 141-147.

Domínguez, J.M., Crespo, A.J.C, Valdez-Balderas, D., Rogers, B.D. \& Gómez-Gesteira, M. [2013] "New multi-GPU implementation for smoothed particle hydrodynamics on heterogeneous clusters," Computer Physics Communications 184(8), 1848-1860.

Duan, G. Chen, B., Zhang, X. \& Wang, Y. [2017] “A multiphase MPS solver for modeling multi-fluid interaction with free surface and its application in oil spill," Computer Methods in Applied Mechanics and Engineering 320, 133-161.

Farahani, R.J. \& Dalrymple, R.A. [2014] “Three-dimensional reversed horseshoe vortex structures under broken solitary waves," Coastal Engineering 91, 261-279.

Farahani, R.J., Dalrymple, R.A., Hérault, A. \& Bilotta, G. [2014] "Three-Dimensional SPH Modeling of a Bar/Rip Channel System," Journal of Waterway, Port, Coastal, and Ocean Engineering 140(1), 82-99. 
Fernandez-Gutierrez, D., Souto-Iglesias, A. \& Zohdi, T.I. [2017] “A hybrid Lagrangian Voronoi-SPH scheme," Proceedings of SPHERIC 2017, $12^{\text {th }}$ international SPHERIC workshop, Ourense, Spain, June 13-15.

Ferrand, M., Laurence D., Rogers, B.D., Violeau, D. \& Kassiotis, C. [2012] "Unified semi-analytical wall boundary conditions for inviscid, laminar or turbulent flows in the meshless SPH method," International Journal for Numerical Methods in Fluids 71(4), $446-472$.

Ferrand, M., Joly, A., Kassiotis, C, Violeau, D., Leroy, A., Morel, F.X. \& Rogers, B.D. [2017] "Unsteady open boundaries for SPH using semi-analytical conditions and Riemann solver in 2D," Computer Physics Communications 210, 29-44.

Foias, C., Manley, O., Rosa, R. \& Temam, R. [2001] "Navier-Stokes Equations and Turbulence," Cambridge University Press, pp. 364.

Fourey, G., Oger, G., Le Touzé, D. \& Alessandrini, B. [2010] "Violent Fluid-Structure Interaction simulations using a coupled SPH/FEM method," IOP Conference Series: Materials Science and Engineering 10(1).

Fourey, G., Hermange, C., Le Touzé, D. \& Oger, G. [2017] “An efficient FSI coupling strategy between Smoothed Particle Hydrodynamics and Finite Element methods," Computer Physics Communications 217, 66-81.

Fourtakas, G. \& Rogers, B.D. [2016] "Modelling multi-phase liquid-sediment scour and resuspension induced by rapid flows using Smoothed Particle Hydrodynamics (SPH) accelerated with a Graphics Processing Unit (GPU)," Advances in Water Resources 92, 186-199.

Fourtakas, G., Stansby, P.K., Rogers, B.D., Lind, S.J., Yan, S. \& Ma, Q.W. [2017] “On the 
coupling of Incompressible SPH with a Finite Element potential flow solver for nonlinear free surface flows," Proceedings of the Twenty-seventh International Ocean and Polar Engineering Conference (ISOPE), San Francisco, CA, USA, June 25-30.

Fourtakas, G., Stansby, P.K., Rogers, B.D. \& Lind, S.J. [2018] “An Eulerian-Lagrangian incompressible SPH formulation (ELI-SPH) connected with a sharp interface," Computer Methods in Applied Mechanics and Engineering 329, 532-552.

Gao, R., Ren, B., Wang, G.Y. \& Wang, Y.X. [2012] "Numerical modelling of regular wave slamming on subface of open-piled structures with the corrected SPH method," Applied Ocean Research 34, 173-186.

Ghaïtanellis, A., Violeau, D., Ferrand, M., Abderrezzak, K.E.K., Leroy, A. \& Joly, A. [2018] "A SPH elastic-viscoplastic model for granular flows and bed-load transport," Advances in Water Resources 111, 156-173.

Gingold, R.A. \& Monaghan, J.J. [1977] "Smoothed particle hydrodynamics: theory and application to non-spherical stars," Monthly Notices of the Royal Astronomical Society $181,375-89$.

Gómez-Gesteira, M., Cerqueiro, D., Crespo, C. \& Dalrymple, R.A. [2005] “Green water overtopping analyzed with a SPH model," Ocean Engineering 32, 223-238.

Gómez-Gesteira, M., Rogers, B.D., Crespo, A.J.C., Dalrymple, R.A., Narayanaswamy, M. \& Dominguez, J.M. [2012a] "SPHysics-development of a free-surface fluid solver-Part 1: Theory and formulations," Computers \& Geosciences 48, 289-299. 
Gómez-Gesteira, M., Crespo, A.J.C., Rogers, B.D., Dalrymple, R.A., Dominguez, J.M. \& Barreiro, A. [2012b] "SPHysics-development of a free-surface fluid solver-Part 2: Efficiency and test cases," Computers \& Geosciences 48, 300-307.

Gong, K., Shao, S., Liu, H., Wang, B. \& Tan, S.K. [2016] “Two-phase SPH simulation of fluid -structure interactions," Journal of Fluids and Structures 65, 155-179.

Gotoh, H., Shibahara, T. \& Sakai, T. [2001] "Sub-Particle-Scale Turbulence Model for the MPS Method-Lagrangian Flow Model for Hydraulic Engineering," Computational Fluid Dynamics Journal 9(4), 339-347.

Gotoh, H., Khayyer, A., Ikari, H., Arikawa, T. \& Shimosako, K. [2014] “On enhancement of Incompressible SPH method for simulation of violent sloshing flows," Applied Ocean Research 46, 104-105.

Gotoh, H. \& Khayyer, A. [2016] "Current achievements and future perspectives for projection-based particle methods with applications in ocean engineering," Journal of Ocean Engineering and Marine Energy 2, 251-278.

Gotoh, H. \& Okayasu, A. [2017] "Computational wave dynamics for innovative design of coastal structures," Proceedings of the Japan Academy, Ser. B 93(9), 525-546.

Gotoh, H. \& Sakai, T. [2006] "Key issues in the particle method for computation of wave breaking," Coastal Engineering 53(2), 171-179.

Gui, Q., Dong, P., Shao, S. \& Chen, Y. [2015] "Incompressible SPH simulation of wave interaction with porous structure," Ocean Engineering 110, 126-139. 
Harada E., Gotoh H., Ikari, H. \& Khayyer, A. [2017] "Numerical simulation for sediment transport using MPS-DEM coupling model," Advances in Water Resources, in press DOI: https://doi.org/10.1016/j.advwatres.2017.08.007

Harada, E., Ikari, H., Shimizu, Y., Khayyer, A. \& Gotoh, H. [2018] "Numerical investigation of the morphological dynamics of a step and pool riverbed using DEM-MPS," Journal of Hydraulic Engineering 144(1), 04017058.

Hérault, A., Billotta, G. \& Dalrymple, R.A. [2010] "SPH on GPU with CUDA," Journal of Hydraulic Research 48, 74-79.

Hori, C., Gotoh, H., Ikari, H. \& Khayyer, A. [2011] “GPU-acceleration for Moving Particle Semi-implicit method," Computers and Fluids 51(1), 174-183.

Hu, F., Matsunaga, T., Tamai, T. \& Koshizuka, S. [2017a] “An ALE particle method using upwind interpolation," Computers and Fluids 145, 21-36.

Hu, W., Pan, W., Rakhsha, M., Tian, Q., Hu, H. \& Negrut, D. [2017b] “A consistent multi resolution smoothed particle hydrodynamics method," Computer Methods in Applied Mechanics and Engineering 324, 278-299.

Hwang, S.C., Khayyer, A., Gotoh, H. \& Park, J.C. [2014] "Development of a fully Lagrangian MPS-based coupled method for simulation of fluid-structure interaction problems," Journal of Fluids and Structures 50, 497-511.

Ikari, H., Gotoh, H. \& Arai, T. [2010] "Fluid-Elastoplastic Hybrid Model for computational mechanics of wave-induced sea cliff erosion," Journal of Japan Society of Civil Engineers, Ser. B2 (Coastal Engineering) 66(1), 916-920 (in Japanese) 
Ikari, H., Gotoh, H., Tanbo, T. \& Ejiri, T. [2015] "MPS-based simulation of scouring due to submerged vertical jet with sub-particle-scale suspended sediment model," Journal of Japan Society of Civil Engineers, Ser. B2 (Coastal Engineering) 71, 19-24. (in Japanese)

Ikari, H., Gotoh, H., Tsuruta, N. \& Kobayashi, Y. [2017] "Wave overtopping analysis on wave absorbing seawall using improved multi-resolution MPS method," Journal of Japan Society of Civil Engineers, Ser. B2 (Coastal Engineering) 73(2), 19-24. (in Japanese)

Inutsuka, S. [2002] "Reformulation of Smoothed Particle Hydrodynamics with Riemann Solver," Journal of Computational Physics 179, 238-267.

Issa, R., Violeau, D., Lee, E.S. \& Flament, H. [2010] "Modelling nonlinear water waves with RANS and LES SPH models," in Advances in Numerical Simulation of Nonlinear Water Waves, vol. 11. Editor, Q. W. Ma, World Scientific Publishing Co, ch. 14.

Jian, W., Liang, D., Shao, S., Chen, R. \& Yang, K. [2016] "Smoothed Particle Hydrodynamics simulations of dam-break flows around movable structures," International Journal of Offshore and Polar Engineering 26(1), 33-40.

Kakuda, K., Nagashima, T., Hayashi, Y., Obara, S., Toyotani, J., Miura, S. \& Matsuda, S. [2013] "Three-dimensional fluid flow simulations using GPU-based particle method," Computer Modeling in Engineering \& Sciences 93(5), 363-376.

Kazemi, E., Nichols, A., Tait, S. \& Shao, S. [2017] "SPH modelling of depth-limited turbulent open channel flows over rough boundaries," International Journal for Numerical Methods in Fluids 83, 3-27. 
Khayyer, A., Gotoh, H. \& Shimizu, Y. [2017a] "Comparative study on accuracy and conservation properties of two particle regularization schemes and proposal of an optimized particle shifting scheme in ISPH context," Journal of Computational Physics 332, 236-256.

Khayyer, A., Gotoh, H., Shimizu, Y. \& Gotoh, K. [2017b] “On enhancement of energy conservation properties of projection-based particle methods," European Journal of Mechanics B/Fluids 66, 20-37.

Khayyer, A., Gotoh, H., Shimizu, Y., Gotoh, K. \& Shao, S. [2017c] “An enhanced particle method for simulation of fluid flow interactions with saturated porous media," Journal of Japan Society of Civil Engineers, Ser. B2 (Coastal Engineering) 73(2), 841-846.

Khayyer, A., Gotoh, H, Falahaty, H., Shimizu, Y. \& Nishijima, Y. [2017d] "Towards development of a reliable fully-Lagrangian MPS-based FSI solver for simulation of 2D Hydroelastic slamming," Ocean Systems Engineering 7(3), 299-318.

Khayyer, A., Gotoh, H., Shimizu, Y. \& Falahaty, H. [2017e] "An Enhanced ISPH-SPH Coupled Method for Incompressible Fluid-Elastic Structure Interactions," Advances of Smoothed Particle Hydrodynamics: the Proceedings of the 2017 SPHERIC Beijing International Workshop, 23-29.

Khayyer, A. \& Gotoh, H. [2016] “A multiphase compressible-incompressible particle method for water slamming," International Journal of Offshore and Polar Engineering 26(1), 20-25.

Koshizuka, S. \& Oka, Y. [1996] "Moving particle semi-implicit method for fragmentation of incompressible fluid," Nuclear Science and Engineering 123, 421-434. 
Koshizuka, S. [2011] "Current achievements and future perspectives on particle simulation technologies for fluid dynamics and heat transfer," Journal of Nuclear Science and Technology 48(2), 155-168.

Le Touzé, D., Marsh, A., Oger, G., Guilcher, P.M., Khaddaj-Mallat, C., Alessandrini, B. \& Ferrant, P. [2010] "SPH simulation of green water and ship flooding scenarios," Journal of Hydrodynamics, Ser. B 22(5) 231-236.

Le Touzé, D., Colagrossi, A., Colicchio, G. \& Greco, M. [2013] "A critical investigation of smoothed particle hydrodynamics applied to problems with free surfaces," International Journal for Numerical Methods in Fluids 73, 660-691.

Lee, E.S., Moulinec, C., Xu, R., Violeau, D., Laurence, D. \& Stansby, P.K. [2008] "Comparisons of weakly compressible and truly incompressible algorithms for the SPH mesh free particle method," Journal of Computational Physics 227(18), 8417-8436.

Leroy, A., Violeau, D., Ferrand, M. \& Joly, A. [2015] "Buoyancy modelling with incompressible SPH for laminar and turbulent flows," International Journal for Numerical Methods in Fluids 78(8), 455-474.

Li, J., Liu, X., Gong, K., Tan, S.K. \& Shao, S. [2012] "SPH modeling of solitary wave fissions over uneven bottoms," Coastal Engineering 60, 261-275.

Liang, D., Jian, W., Shao, S., Chen, R. \& Yang, K. [2017] “Incompressible SPH simulation of solitary wave interaction with movable seawalls," Journal of Fluids and Structures 69, 72-88.

Lind, S.J., Xu, R., Stansby, P.K. \& Rogers, B.D. [2012] "Incompressible smoothed particle hydrodynamics for free-surface flows: a generalised diffusion-based algorithm for 
stability and validations for impulsive flows and propagating waves," Journal of Computational Physics 231(4), 1499-1523.

Lind, S.J., Stansby, P.K., Rogers, B.D. \& Lloyd, P.M. [2015] "Numerical predictions of water-air wave slam using incompressible-compressible smoothed particle hydrodynamics," Applied Ocean Research 49, 57-71.

Lind, S.J., Stansby, P.K. \& Rogers, B.D. [2016a] "Fixed and moored bodies in steep and breaking waves using SPH with the Froude-Krylov approximation," Journal of Ocean Engineering and Marine Energy 2, 331-354.

Lind, S.J., Stansby, P.K. \& Rogers, B.D. [2016b] "Incompressible-compressible flows with a transient discontinuous interface using smoothed particle hydrodynamics (SPH)," Journal of Computational Physics 309, 129-147.

Lind, S.J., Fang, Q., Stansby, P.K., Rogers, B.D. \& Fourtakas, G. [2017] "A two-phase incompressible-compressible (water-air) Smoothed Particle Hydrodynamics (ICSPH) method applied to focused wave slam on decks," Proceedings of the Twenty-seventh International Ocean and Polar Engineering Conference (ISOPE), San Francisco, CA, USA, June 25-30.

Lind, S.J. \& Stansby, P.K. [2015] "Investigations into higher order incompressible SPH," Proc. $10^{\text {th }}$ SPHERIC Int. Workshop, Parma, Italy, 131-138.

Lind, S.J. \& Stansby, P.K. [2016] "High-order Eulerian incompressible smoothed particle hydrodynamics with transition to Lagrangian free-surface motion," Journal of Computational Physics 326, 290-311. 
Litvinov, S., Hu, X.Y. \& Adams, N.A. [2015] "Towards consistence and convergence of conservative SPH approximations," Journal of Computational Physics 301, 394-401.

Liu, M.B. \& Liu, G.R. [2015] "Particle Methods for Multi-Scale and Multi-Physics," World Scientific Publishing Co., ISBN: 978-9814571692, pp. 376.

Ma, H., Mizutani, N., Eguchi, S. \& Hur, D. [2004] "Study on beach profile change and wave induced velocity field in permeable beach," Proceedings of Civil Engineering in Ocean 20, 509-514 (in Japanese).

Makris, C.V., Memos, C.D. \& Kresteniti, Y.N. [2016] "Numerical modeling of surfzone dynamics under weakly plunging breakers with SPH method," Ocean Modelling 98, $12-35$.

Marrone, S., Antuono, M., Colagrossi, A., Colicchio, G., Le Touzé, D. \& Graziani, G. [2011] " $\delta$-SPH model for simulating violent impact flows," Computer Methods in Applied Mechanics and Engineering 200, 1526-1542.

Marrone, S., Bouscasse, B., Colagrossi, A. \& Antuono, M. [2012] "Study of ship wave breaking patterns using 3D parallel SPH simulations," Computers \& Fluids 69, 54-66.

Marrone, S., Colagrossi, A., Di-Mascio, A. \& Le Touzé, D. [2015] "Prediction of energy losses in water impacts using incompressible and weakly compressible models," Journal of Fluids and Structures 54, 802-822.

Marrone, S., Di Mascio, A. \& Le Touzé, D. [2016] “Coupling of Smoothed Particle Hydrodynamics with Finite Volume method for free-surface flows," Journal of Computational Physics 310, 161-180. 
Marrone, S., Colagrossi, A., Park, J.S. \& Campana, E.F. [2017] "Challenges on the numerical prediction of slamming loads on LNG tank insulation panels," Ocean Engineering 141, 512-530.

Mayrhofer, A., Laurence, D., Rogers, B.D. \& Violeau, D. [2015] "DNS and LES of 3-D wall-bounded turbulence using Smoothed Particle Hydrodynamics," Computers \& Fluids 115, 86-97.

Meringolo, D.D., Colagrossi, A., Marrone, S. \& Aristodemo, F. [2017] “'On the filtering of acoustic components in weakly-compressible SPH simulations," Journal of Fluids and Structures 70, 1-23.

Mokos, A., Rogers, B.D. \& Stansby, P.K. [2017] “A multi-phase particle shifting algorithm for SPH simulations of violent hydrodynamics with a large number of particles," Journal of Hydraulic Research 55(2) 143-162.

Monaghan, J.J. [2012] "Smoothed particle hydrodynamics and its diverse applications," Annual Review of Fluid Mechanics 44(1), 323-346 .

Monaghan, J.J. \& Rafiee, A. [2013] “A simple SPH algorithm for multi-fluid flow with high density ratios," International Journal for Numerical Methods in Fluids 71, $537-561$.

Mori, N. \& Kakuno, S. [2008] “Aeration and bubble measurements of coastal breaking waves," Fluid Dynamics Research 40(7-8), 616-626.

Oger, G., Guilcher, P.M., Jacquin, E., Brosset, L., Deuff, J.B. \& Le Touzé, D. [2010] "Simulations of hydro-elastic impacts using a parallel SPH model," International Journal of Offshore and Polar Engineering 20(3), 181-189. 
Oger, G., Le Touzé, D., Guibert, D., de Leffe, M., Biddiscombe, J., Soumagne, J. \& Piccinali, J.G. [2016a] “On distributed memory MPI-based parallelization of SPH codes in massive HPC context," Computer Physics Communications 200, 1-14.

Oger, G., Marrone, S., Le Touzé, D. \& de Leffe, M. [2016b] “SPH accuracy improvement through the combination of a quasi-Lagrangian shifting transport velocity and consistent ALE formalisms," Journal of Computational Physics 313, 76-98.

Ovaysi, S. \& Piri, M [2012] "Multi-GPU acceleration of direct pore-scale modeling of fluid flow in natural porous media," Computer Physics Communications 183, 1890-1898.

Peng, C., Xu, G., Wu, W., Yu, H. \& Wang, C. [2017] "Multiphase SPH modeling of free

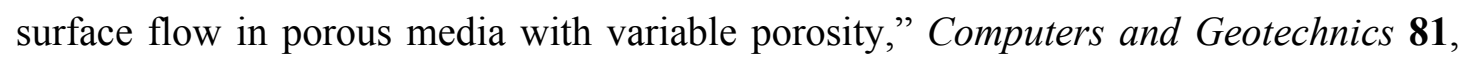
$239-248$.

Qiu, L.C. [2014] "OpenCL-based GPU acceleration of ISPH simulation for incompressible flows," In Applied Mechanics and Materials 444, 380-384.

Rafiee, A. \& Thiagarajan, K.P. [2009] "An SPH projection method for simulating fluid-hypoelastic structure interaction," Computer Methods in Application Mechanics and Engineering 198, 2785-2795.

Rafiee, A., Cummins, S., Rudman, M. \& Thiagarajan, K. [2012] "Comparative study on the accuracy and stability of SPH schemes in simulating energetic free-surface flows," European Journal of Mechanics - B/Fluids 36, 1-16.

Ran, Q., Tong, J., Shao, S., Fu, X. \& Xu, Y. [2015] "Incompressible SPH scour model for movable bed dam break flows," Advances in Water Resources 82, 39-50. 
Razavitoosi, S.L., Ayyoubzadeh, S.A. \& Valizadeh, A. [2014] “Two-phase SPH modelling of waves caused by dam break over a movable bed," International Journal of Sediment Research 29(3), 344-356.

Ren, B., Jin, Z, Gao, R., Wang, Y.X. \& Xu, Z.L. [2013] "SPH-DEM modeling of the hydraulic stability of 2D blocks on a slope," Journal of Waterway, Port, Coastal, and Ocean Engineering 140(6), 04014022.1-04014022.12.

Ren, B., Wen, H., Dong, P. \& Wang, Y. [2014] "Numerical simulation of wave interaction with porous structures using an improved smoothed particle hydrodynamic method," Coastal Engineering 88, 88-100.

Ren, B., He, M., Dong, P. \& Wen, H. [2015] "Nonlinear simulations of wave-induced motions of a freely floating body using WCSPH method," Applied Ocean Research 50, $1-12$.

Ren, B., He, M., Li, Y. \& Dong, P. [2017] "Application of smoothed particle hydrodynamics for modeling the wave-moored floating breakwater interaction," Applied Ocean Research 67, 277-290.

Shadloo, M.S., Weiss, R., Yildiz, M. \& Dalrymple, R.A. [2015] "Numerical simulation of long wave runup for breaking and nonbreaking waves," International Journal of Offshore and Polar Engineering 25(1), 1-7.

Shadloo, M.S., Oger, G. \& Le Touzé, D. [2016] "Smoothed particle hydrodynamics method for fluid flows, towards industrial applications: Motivations, current state, and challenges," Computers \& Fluids 136, 11-34. 
Shao, S., Ji, C., Graham, D.I., Reeve, D.E., James, P.W. \& Chadwick, A.J. [2006] "Simulation of wave overtopping by an incompressible SPH model," Coastal Engineering 53(9), 723-735.

Shao, S. \& Lo, E.Y.M. [2003] "Incompressible SPH method for simulating Newtonian and non-Newtonian flows with a free surface," Advances in Water Resources 26, 787-800.

Shao, S. [2010] "Incompressible SPH flow model for wave interactions with porous media," Coastal Engineering 57, 304-316.

Shi, H., Yu, X. \& Dalrymple, R.A. [2017] "Development of a two-phase SPH model for sediment laden flows," Computer Physics Communications 221, 259-272.

Shi, Y., Li, S., Chen, H., He, M. \& Shao, S. [2018] "Improved SPH simulation of spilled oil contained by flexible floating boom under wave-current coupling condition," Journal of Fluids and Structures 76, 272-300.

Shibata, K., Koshizuka, S., Matsunaga, T. \& Masaie, I. [2017] "The overlapping particle technique for multi-resolution simulation of particle methods," Computer Methods in Applied Mechanics and Engineering 325, 434-464.

Stagonas, D., Warbrick, D., Muller, G. \& Magagna, D. [2011] "Surface tension effects on energy dissipation by small scale, experimental breaking waves," Coastal Engineering 58, $826-836$.

Sun, P.N., Colagrossi, A., Marrone, S. \& Zhang A.M. [2017] "The Splus-SPH model: Simple procedures for a further improvement of the SPH scheme," Computer Methods in Applied Mechanics and Engineering 315, 25-49. 
Sun, P.N., Colagrossi, A., Marrone, S., Antuono, M. \& Zhang A.M. [2018] "Multi-resolution $\delta$ plus-SPH with tensile instability control: Towards high Reynolds number flows," Computer Physics Communications, in press, doi: https://doi.org/10.1016/j.cpc.2017.11.016

Sun, X., Sakai, M. \& Yamada, Y. [2013] "Three-dimensional simulation of a solid-liquid flow by the DEM-SPH method," Journal of Computational Physics 248, 147-176.

Tang Z., Wan D., Chen G. \& Xiao, Q. [2016] "Numerical simulation of 3D violent free-surface flows by multi-resolution MPS method," Journal of Ocean Engineering and Marine Energy 2(3), 355-364.

Tayebi, A. \& Jin, Y.C. [2015] "Development of Moving Particle Explicit (MPE) method for incompressible flows," Computers \& Fluids 117, 1-10.

Tsuruta, N., Khayyer, A. \& Gotoh, H. [2013] "A short note on dynamic stabilization of moving particle semi-implicit method," Computers \& Fluids 82, 158-164.

Tsuruta, N., Khayyer, A. \& Gotoh, H. [2015] "Space potential particles to enhance the stability of projection-based particle methods," International Journal of Computational Fluid Dynamics 29(1), 100-119.

Tsuruta, N., Khayyer, A. \& Gotoh, H. [2016] "A novel refinement technique for projection-based particle methods," Proceedings of SPHERIC 2016, 11 th international SPHERIC workshop, Munich, Germany, June 13-16.

Vacondio, R., Rogers, B.D., Stansby, P.K., Mignosa, P. \& Feldman, J. [2013] "Variable resolution for SPH: A dynamic particle coalescing and splitting scheme," Computer Methods in Applied Mechanics and Engineering 256, 132-148. 
Vacondio, R., Rogers, B.D., Stansby, P.K. \& Mignosa, P. [2016] "Variable resolution for SPH in three dimensions: Towards optimal splitting and coalescing for dynamic adaptivity," Computer Methods in Applied Mechanics and Engineering 300, 442-460.

Violeau, D. [2012] "Fluid Mechanics and the SPH Method, Theory and Applications," Oxford University press, ISBN: 978-0-19-965552-6.

Violeau, D., Buvat, C., Abed-Meraim, K. \& de Nanteuil, E. [2007] "Numerical modelling of boom and oil spill with SPH," Coastal Engineering 54(12), 895-913.

Violeau, D. \& Issa, R. [2007] "Numerical modelling of complex turbulent free-surface flows with the SPH method: an overview," International Journal for Numerical Methods in Fluids 53, 277-304.

Violeau, D. \& Rogers, B.D. [2016] "Smoothed particle hydrodynamics (SPH) for free-surface flows: past, present and future," Journal of Hydraulic Research 54(1), $1-26$.

Wang, D., Li, S., Arikawa, T. \& Gen, H. [2016] "ISPH Simulation of Scour Behind Seawall Due to Continuous Tsunami Overflow," Coastal Engineering Journal 58(3), 1650014.

Wei, Z., Dalrymple, R.A., Hérault, A., Bilotta, G., Rustico, E. \& Yeh, H. [2015] "SPH modeling of dynamic impact of tsunami bore on bridge piers," Coastal Engineering, $104,26-42$.

Wen, H., Ren, B., Dong, P. \& Wang, Y. [2016] “A SPH numerical wave basin for modeling wave-structure interactions," Applied Ocean Research 59, 366-377. 
Xu, H. \& Lin, P. [2017] "A new two-step projection method in an ISPH model for free surface flow computations," Coastal Engineering 127, 68-79.

Xu, R., Stansby, P.K., \& Laurence, D. [2009] “Accuracy and stability in incompressible SPH (ISPH) based on the projection method and a new approach," Journal of Computational Physics 228(18), 6703-6725.

Xu, R. [2010] “An Improved Incompressible Smoothed Particle Hydrodynamics Method and its Application in Free-surface Simulations," P.h.D Thesis, School of Mechanical, Aerospace and Civil Engineering, University of Manchester, United Kingdom.

Xiong, Q., Li, B. \& Xu, J. [2013] “GPU-accelerated adaptive particle splitting and merging in SPH," Computer Physics Communications 184, 1701-1707.

Yeganeh-Bakhtiary, A., Houshangi, H., Hajivalie, F. \& Abolfathi, S. [2017] “A Numerical Study on Hydrodynamics of Standing Waves in Front of Caisson Breakwaters with WCSPH Model, ” Coastal Engineering Journal 59(1), 1750005.

Zhang, C., Hu, X.Y. \& Adams, N.A. [2017] “A weakly compressible SPH method based on a low-dissipation Riemann solver," Journal of Computational Physics 335, 605-620.

Zheng, X., Ma, Q.W. \& Duan, W.Y. [2014] "Comparative study of different SPH schemes on simulating violent water wave impact flows," China Ocean Eng 6(28), 791-806.

Zheng, X., Shao, S., Khayyer, A., Duan, W., Ma, Q. \& Liao, K. [2017a] "Corrected first-order derivative ISPH in water wave simulations," Coastal Engineering Journal 59 (1). 1750010 .

Zheng, X., Lv, X., Ma, Q., Duan, W., Khayyer, A. \& Shao, S. [2017b] “An improved solid boundary treatment for wave-float interactions using ISPH method," International 
Journal of Naval Architecture and Ocean Engineering, in press. DOI: https://doi.org/10.1016/j.ijnaoe.2017.08.001. 

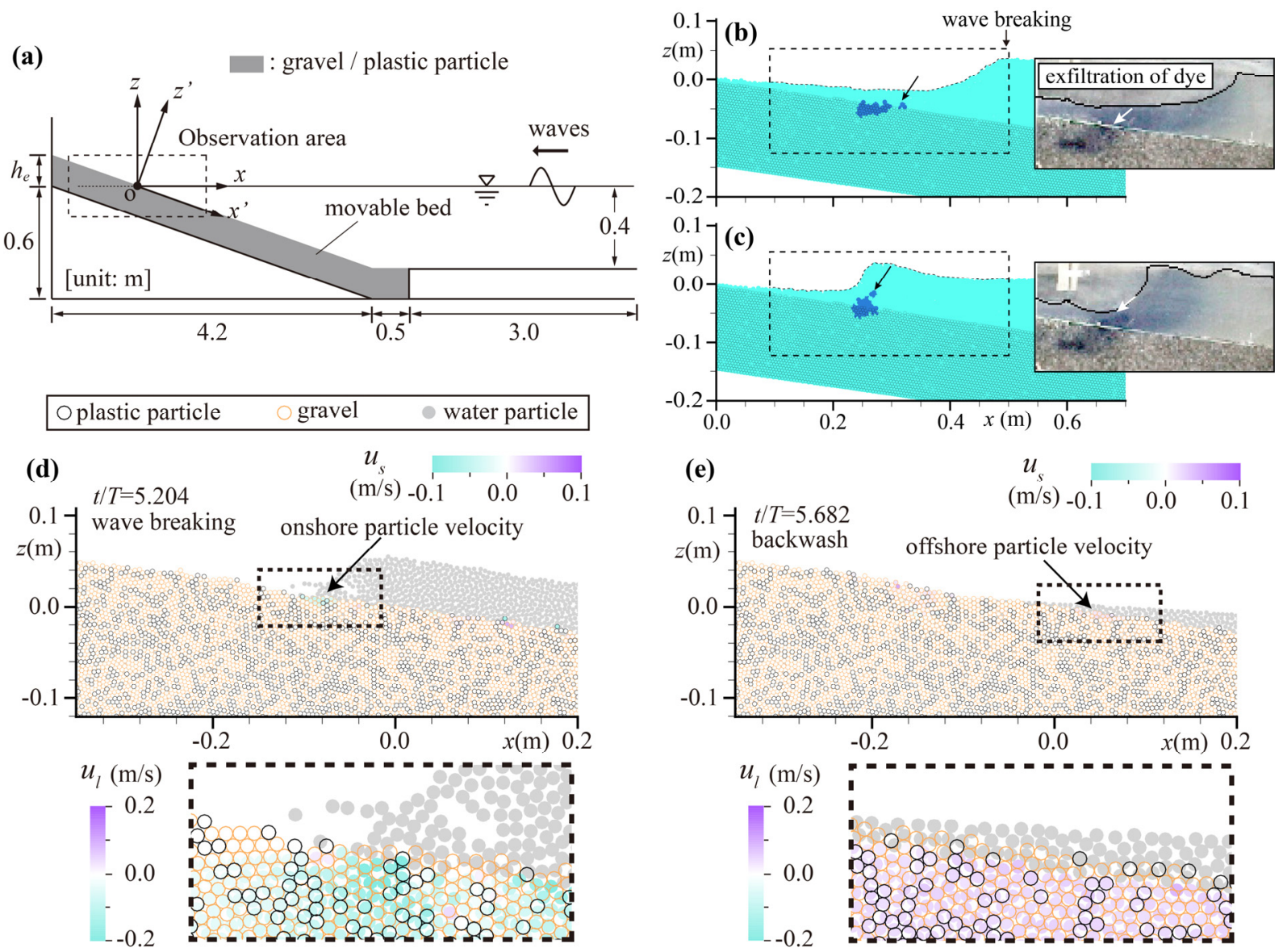

Fig. 1 (a) Computational domain corresponding to the simulation of exfiltration process of dye by DEM-MPS, (b-c) comparisons of exfiltration process between simulation and experiment by Ma et al. (2004), (d-e) particle distributions together with horizontal velocity distributions corresponding to wave breaking and backwash [Harada et al., 2017] 
(a)

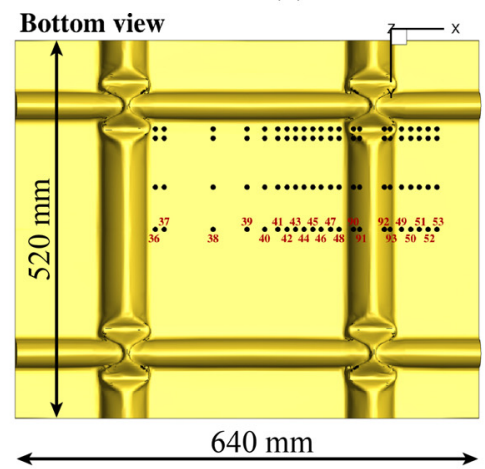

(b)
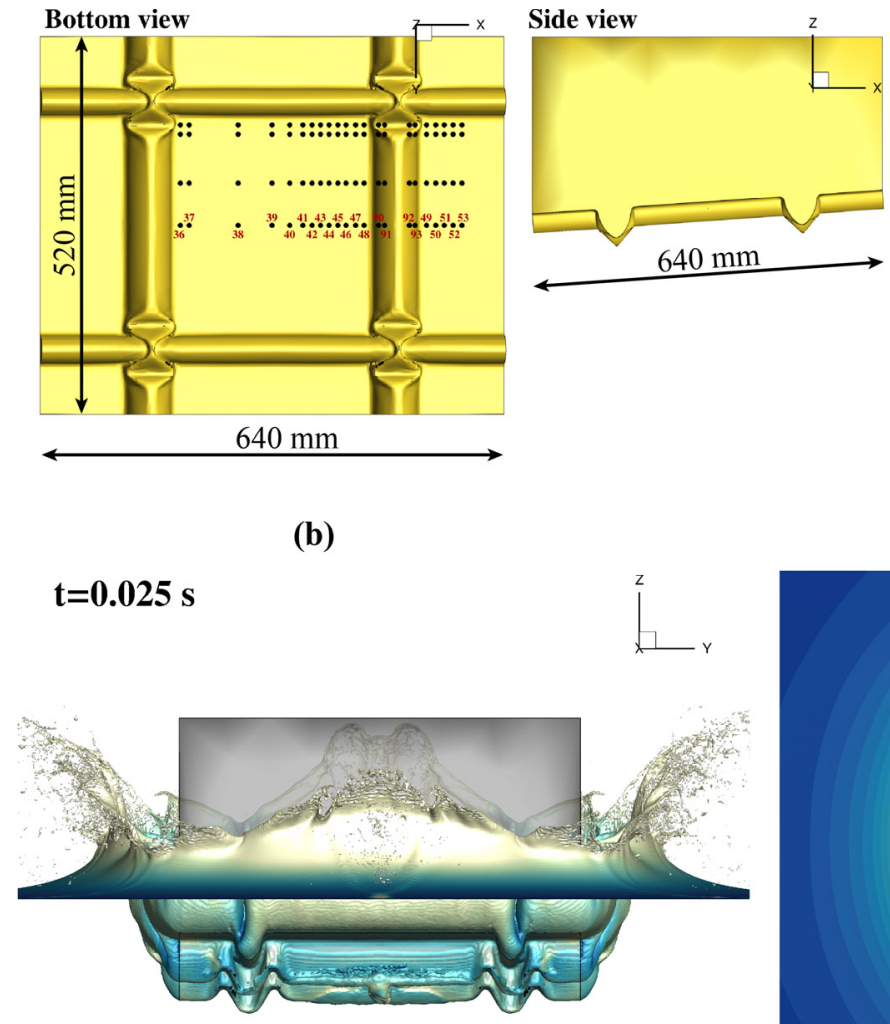

$\mathrm{t}=\mathbf{0 . 0 2 5} \mathrm{s}$
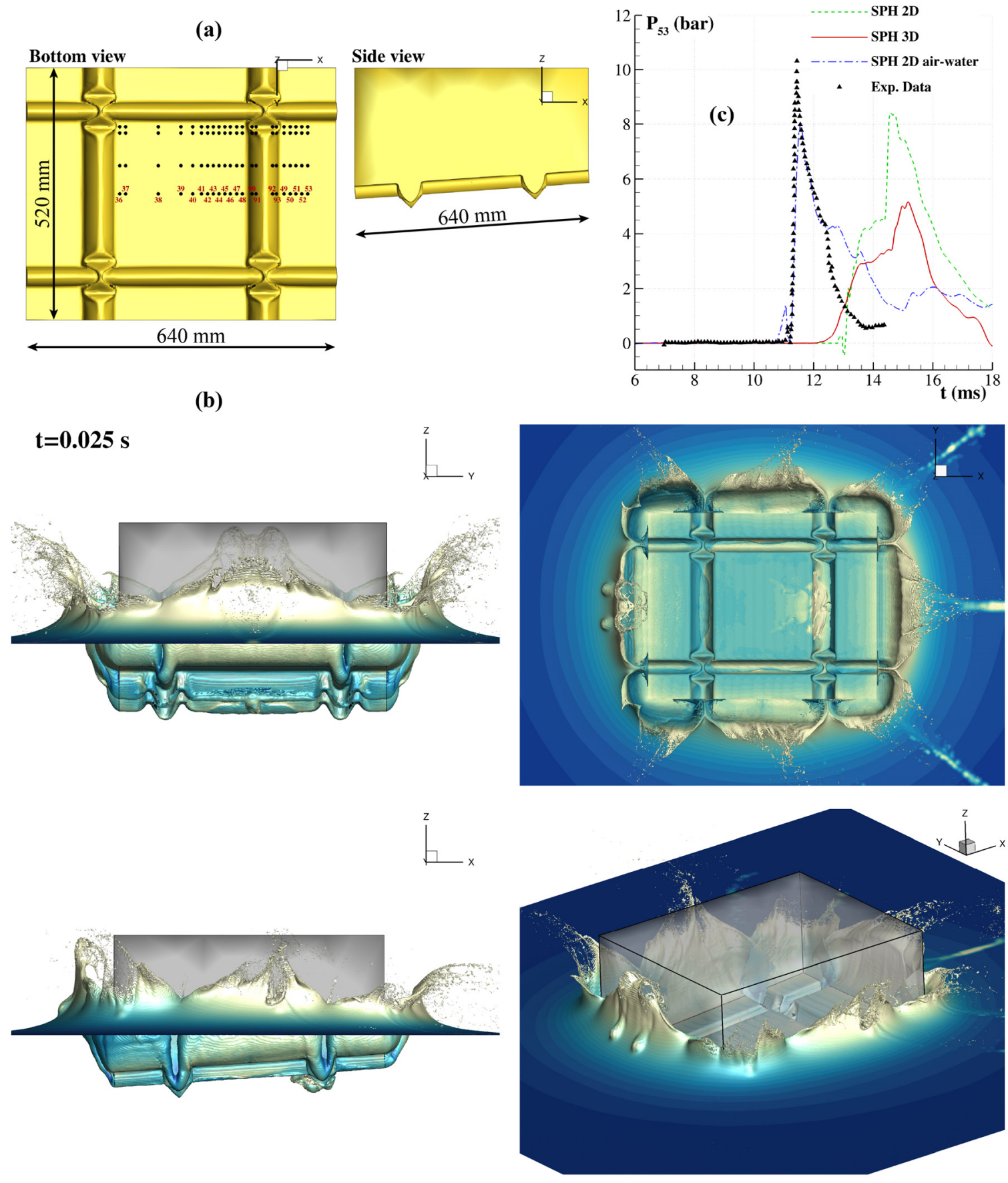

Fig. 2 (a) Schematic side and bottom views of the modeled Mark-III insulation panel along with the position of pressure probes, (b) typical snapshots corresponding to water impact of Mark III type corrugated insulation panel at $t=0.025 \mathrm{~s}$ from different views, (c) time history of pressure at probe P53 obtained from 2D and 3D single-phase SPH, 2D multi-phase SPH vs. experiment [Marrone et al., 2017] 

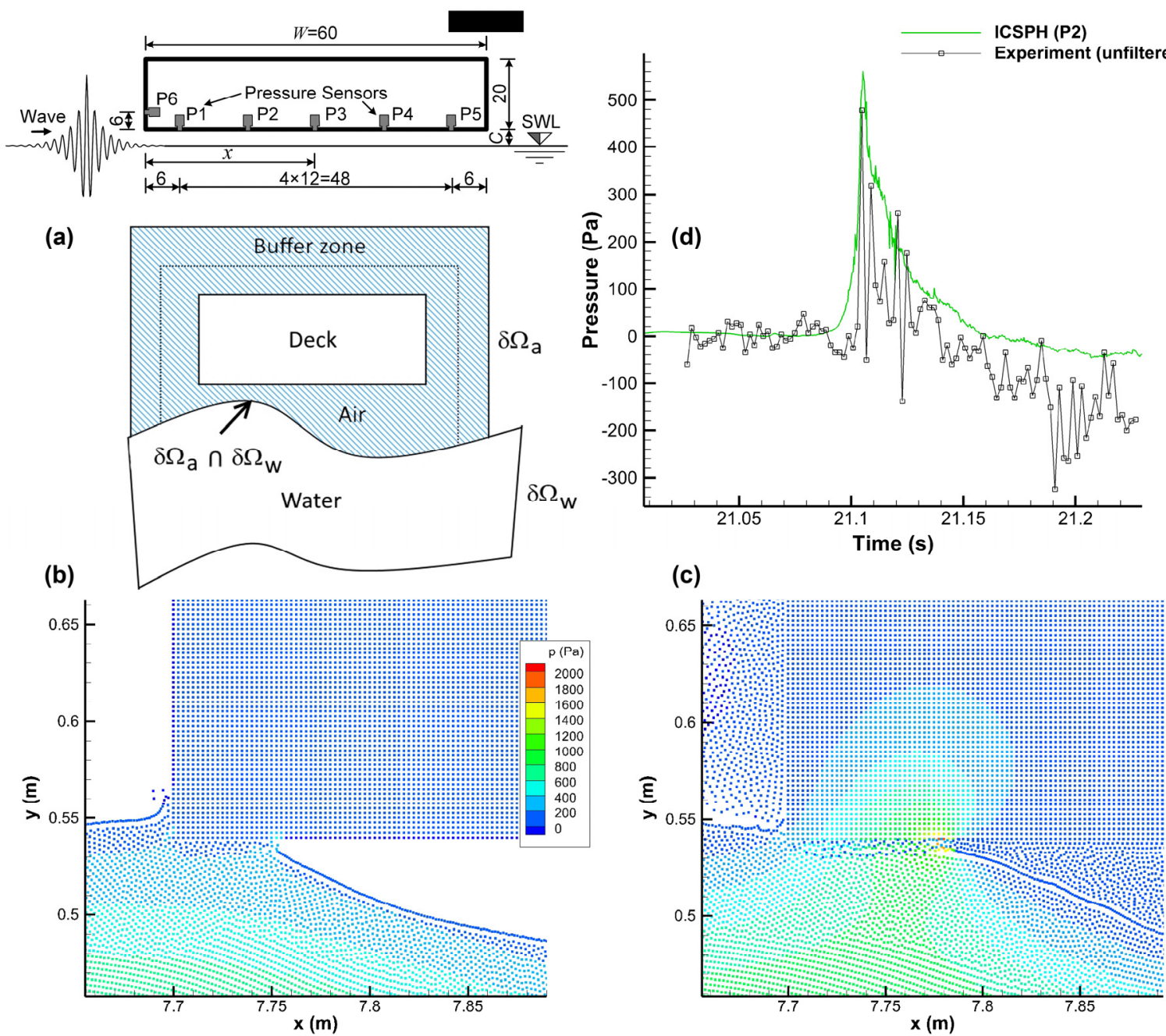

Fig. 3 (a) Schematic sketch of the deck along with the positions of pressure transducers [Lind et al., 2017], (dimensions are in centimeter); (b) and (c) typical snapshots of particle distributions and pressure contours close to $\mathrm{P} 1$ at $t=21.039 \mathrm{~s}$, without and with considering air phase, respectively, (d) time histories of unfiltered pressure measured at P2 in the experiment vs. ICSPH simulation results by Lind et al. [2017] 
(a)

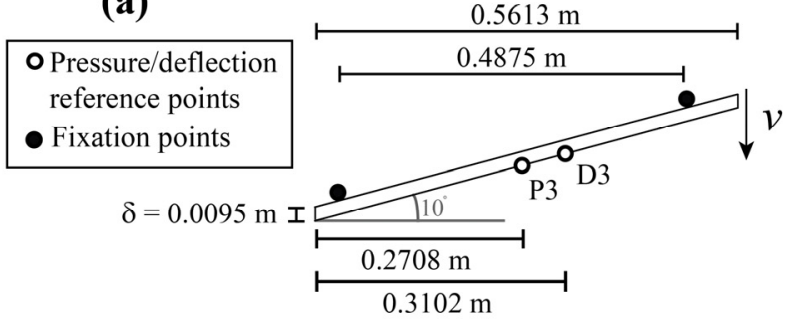

Enhanced MPS-MPS
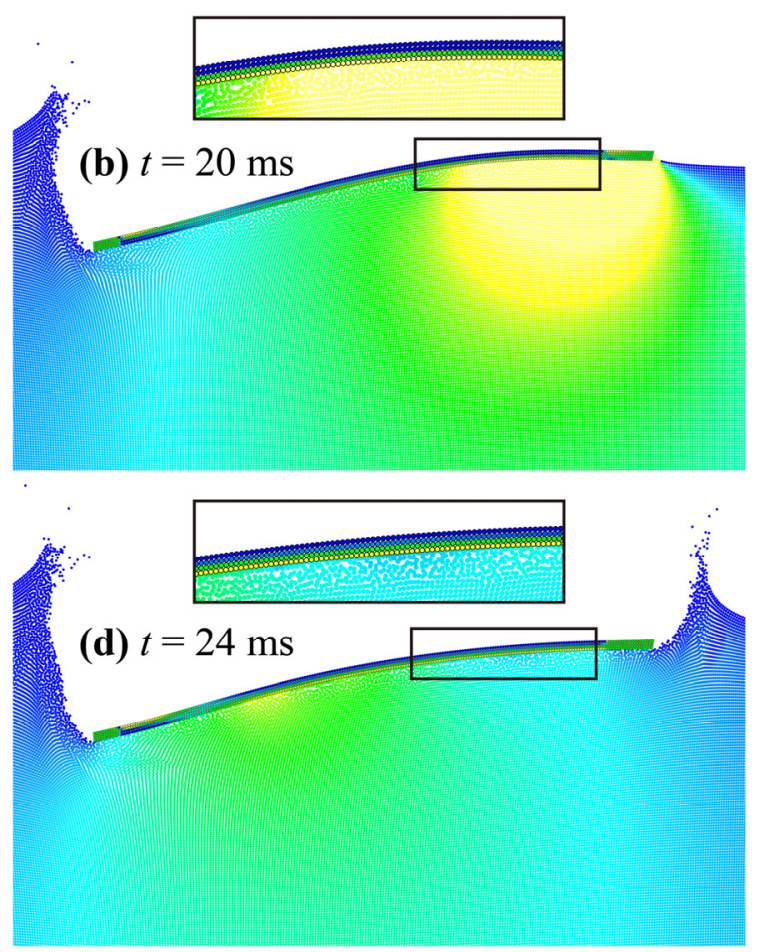

$\sigma_{x x}$

$\left(\mathrm{N} / \mathrm{m}^{2}\right)$

$\begin{array}{lllll}-5 \mathrm{E}+07 & -2.5 \mathrm{E}+07 & 0 & 2.5 \mathrm{E}+07 & 5 \mathrm{E}+07\end{array}$

$p$

$\left(\mathrm{N} / \mathrm{m}^{2}\right)$

$0.0 \mathrm{E}+00 \quad 7.5 \mathrm{E}+04 \quad 1.5 \mathrm{E}+05 \quad 2.2 \mathrm{E}+05 \quad 3.0 \mathrm{E}+05$

\section{Enhanced ISPH-SPH}
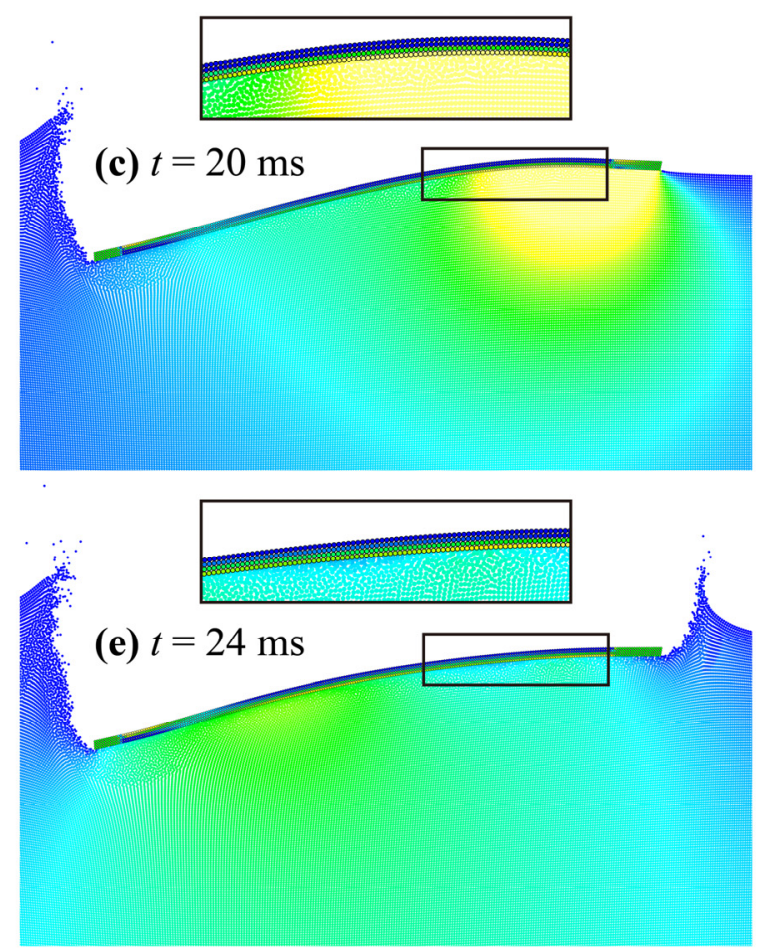

Fig. 4 Qualitative evaluation of the results of simulation by Enhanced MPS-MPS and Enhanced ISPH-SPH in hydrodynamic slamming test of SG panel in case of $v=4 \mathrm{~m} / \mathrm{s}$ [Allen, 2013]; (a) setup of the benchmark test, and typical snapshots illustrating the stress $\left(\sigma_{x x}\right) /$ pressure $(p)$ fields at $t=20 \mathrm{~ms}$ and at $t=24 \mathrm{~ms}$ reproduced by (b,d) Enhanced MPS-MPS [Khayyer et al., 2017d], and (c,e) Enhanced ISPH-SPH [Khayyer et al., 2017e] 

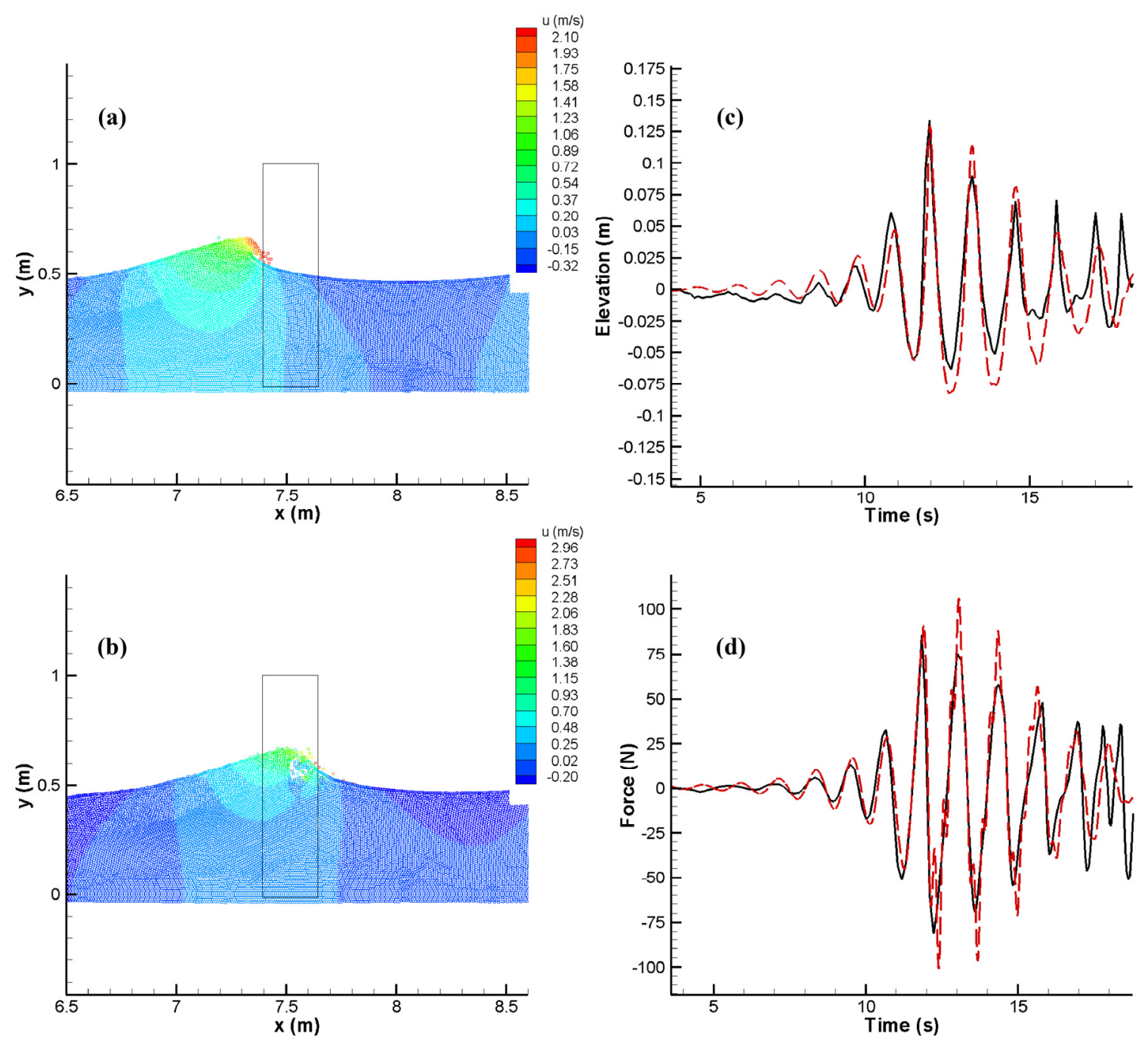

Fig. 5 (a) and (b) typical snapshots showing particle distribution and contours of horizontal velocity at $t=$ $11.83 \mathrm{~s}$ and $t=11.96 \mathrm{~s}$, respectively, obtained from the simulation of plunging wave breaker of focused wave impacting the cylinder, experimental and numerical results corresponding to (c) the free-surface elevation in front of the cylinder and (d) the total horizontal force on the cylinder for focused wave case F15 (the dashed lines denote experimental measurements, the black lines correspond to ISPH) [Lind et al., 2016a] 

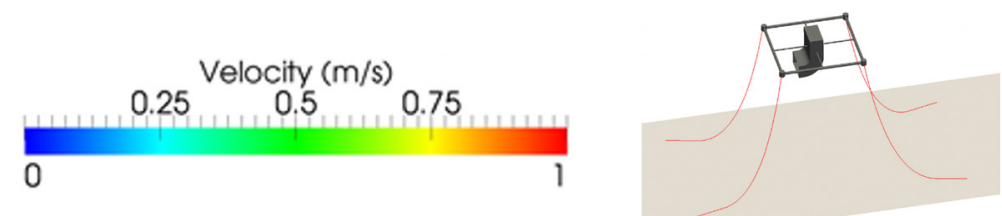

(a)

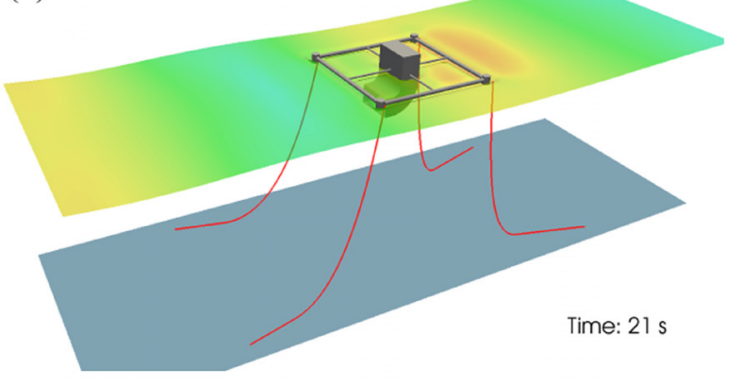

(c)

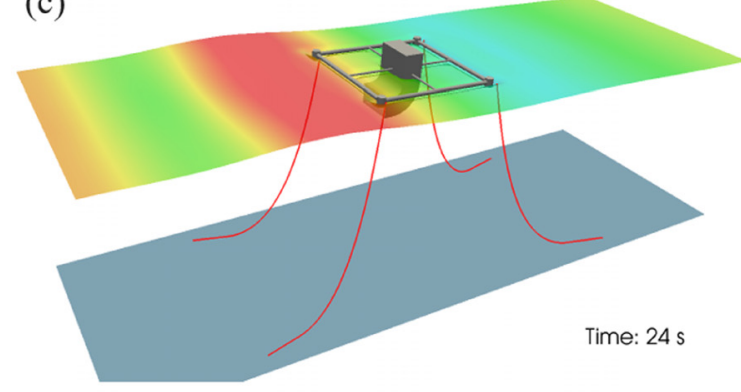

(e)

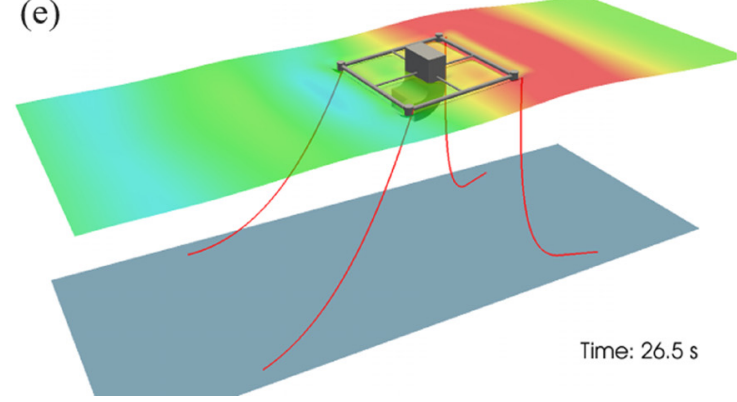

(b)

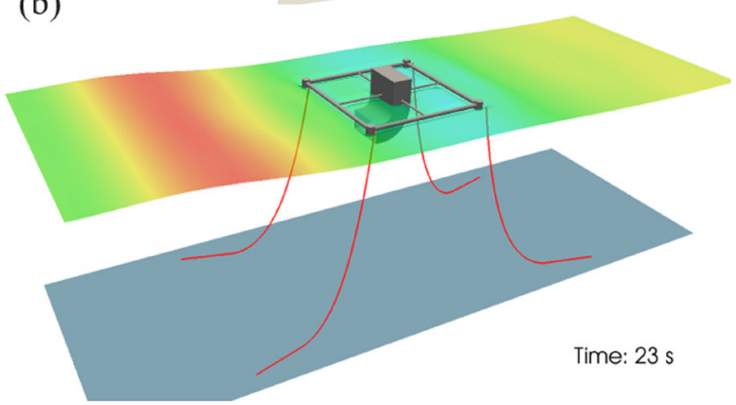

(d)

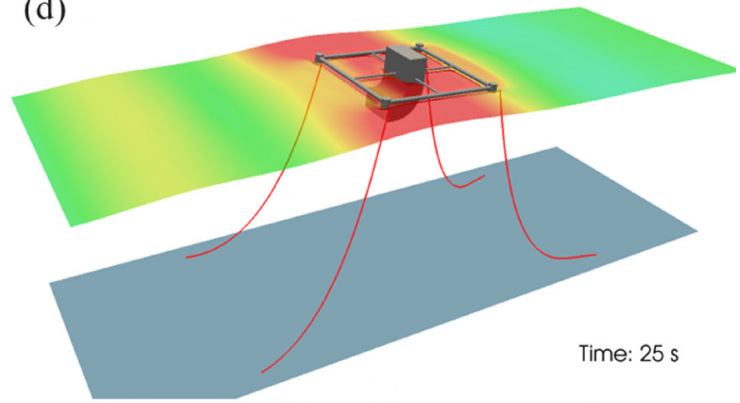

(f)

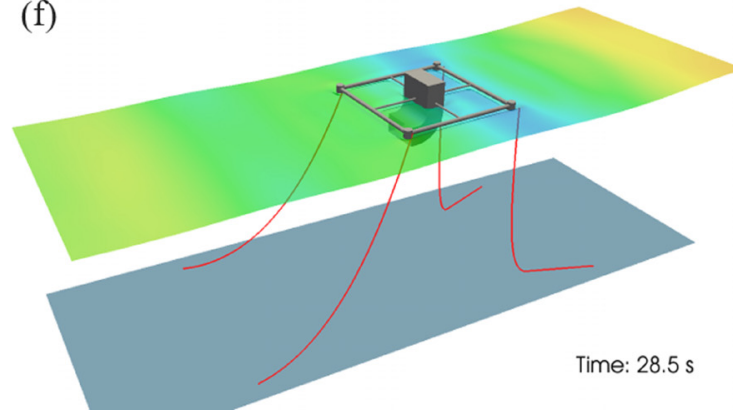

Fig. 6 Typical snapshots corresponding to different instants of the simulation of interaction between sea waves and floating OWC (Oscillating Water Column device) in the open sea [Crespo et al., 2017] 


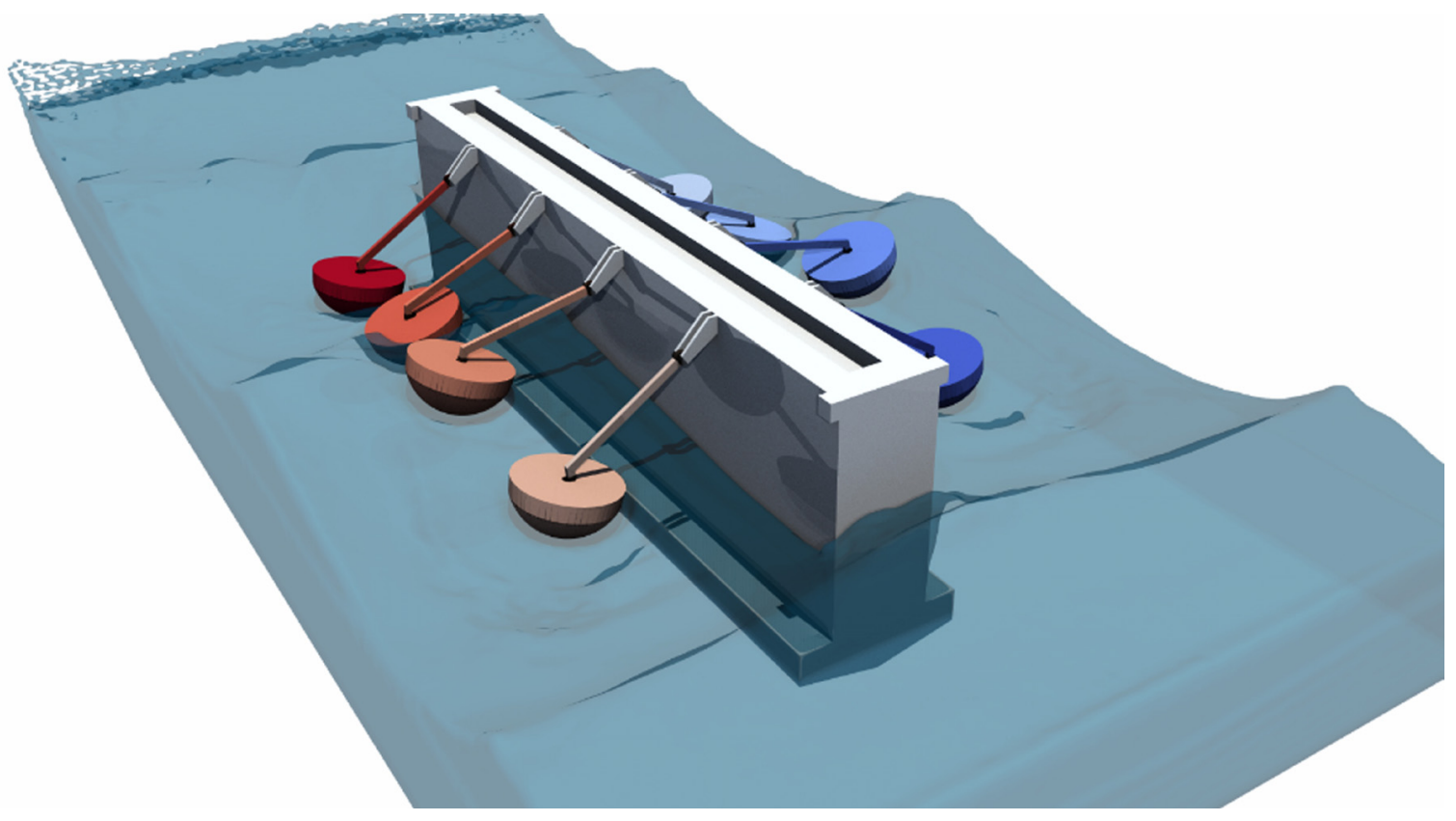

Fig. 7 A Multi-physics SPH-based model representing the system of a Wave Energy Converter (WaveStar) reproduced by DualSPHysics [Canelas et al., 2017a] 

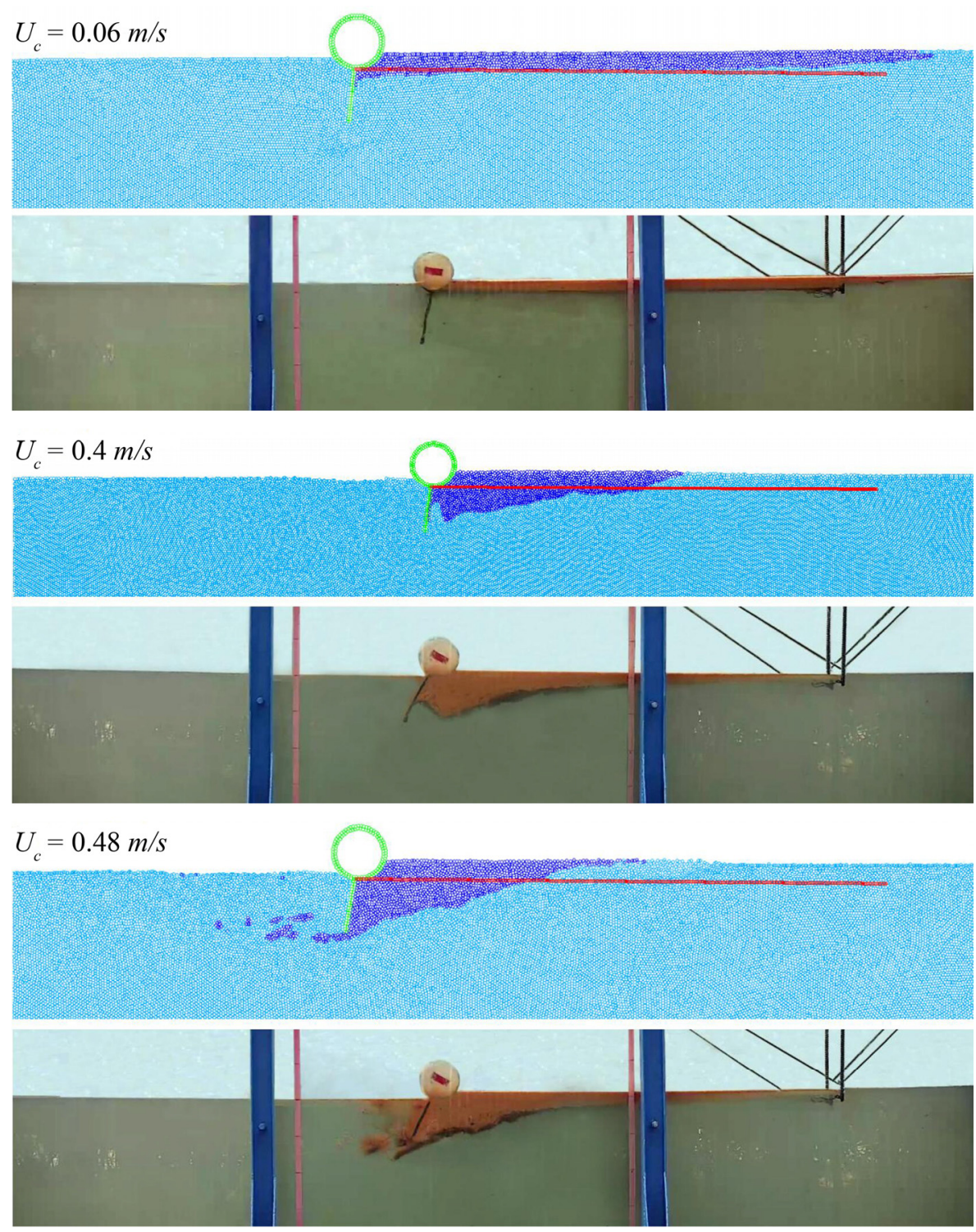

\section{$U_{c}=0.66 \mathrm{~m} / \mathrm{s}$}
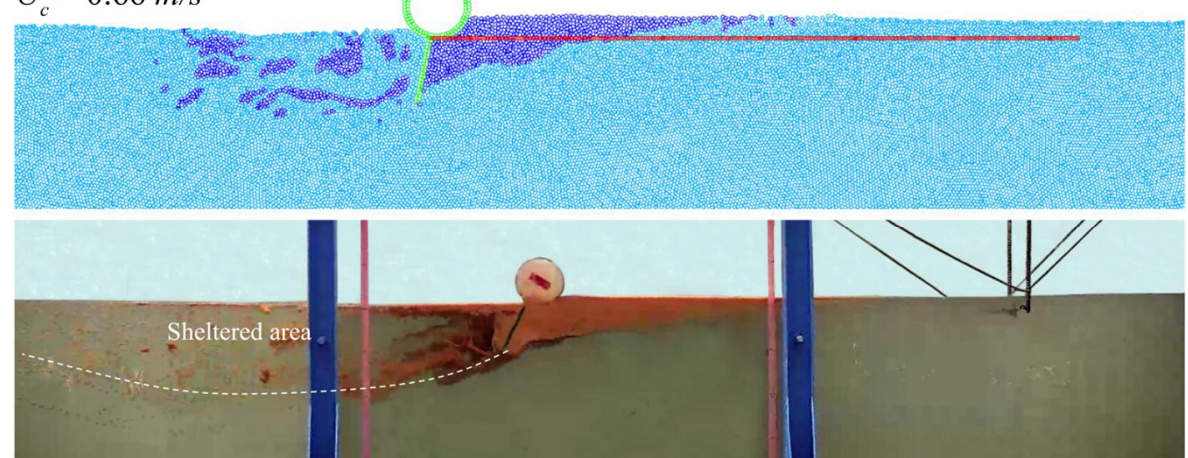

Fig. 8 The evolution process of oil slick contained by a floating boom subjected to currents with different velocities $\left(U_{c}\right)$ - experiments and SPH simulations by Shi et al. [2018] 